\title{
Investigation on the Small Signal Characteristic Based on the LLC Hybrid Hysteretic Charge Control
}

\author{
Richard (Hua) Yang, Brent A. McDonald, and Yalong Li
}

\begin{abstract}
In this paper, an analytical small-signal model applied for hybrid hysteretic charge (HHC) control has been proposed and analyzed with the advantages over direct frequency control (DFC). Based on the approach of extended describing function method and average concept, for the first time, the systematical analytical open loop transfer functions from control to output, input to output, output impedance and the closed transfer functions of the overall loop, audio susceptibility and output impedance are proposed and verified through simulation. Additionally, some important physical insights have been extracted, analyzed and verified. Finally, the experiments on a design example of 12 VDC\&12 A output power are conducted and verified. It shows that the calculations match well with the results from both the simulation and experiment, which reveals the proposed analytical transfer functions are very useful for the practical power design to achieve good prediction result.
\end{abstract}

Index Terms - Analytical small signal model, hybrid hysteretic charge (HHC), hybrid hysteretic charge control, LLC, UCC25630.

\section{INTRODUCTION}

$\mathrm{W}$ ITH the increasing requirement on power efficiency and power density, the LLC topology has become into one of the most popular topologies of the isolated power converters. Numerous articles [1]-[6] have been created to show the best design optimization methods. However, there are the challenges for the conventional DFC LLC converter regarding the control design and loop optimization when input voltage and output voltage have been specified strictly to be within a varied range, in which the task of loop design is hard to be optimized. As for the small signal modeling on PWM converters, the statespace approach based on the average concept proposed by Middlebrook and Cuk [7] is accurate within a limited frequency bandwidth. Later R. Ridley [8] had proposed an improved methodology with the concept of the three-terminal switch, on which the good prediction result in many PWM converters are achieved [9], [10], [11], [12]. In recent years, the advanced describing function method has been successfully applied to most of current control converters with the accurate analytical transfer functions up to the switching frequency in [13]-[14]. However, all these methodologies are hard to obtain a good result if applying to the resonant converter where the switching frequency components and its harmonics have been turned into

Manuscript received April 15, 2019. This paper was presented in part at the 2018 IEEE International Power Electronics and Application Conference and Exposition (PEAC), Shenzhen, China, November 2018.

R. Yang is with Texas Instruments China, Konka R\&D Building, Shenzhen Guangdong 518057, China (email: richard-yang@ti.com)

B. A. McDonald and Y. Li are with Texas Instruments, Dallas, TX, USA.

Digital Object Identifier 10.24295/CPSSTPEA.2019.00013 the dominant variable part and the beat frequency dynamics has occurred. Then the extended describing function method proposed by E. X. Yang [15]-[16] had been successfully applied to the resonant converter to predict the beat frequency dynamic behavior accurately.

To work out the model of LLC converter, lots of researches [17]-[20] based on the extended describing function or other similar averaged modeling concept [21] have been employed and the results are proved to be accurate to predict the beat frequency dynamics. However, the models are still hard to meet the practical engineering design due to its lack of analytical transfer functions for the physical insight extraction and most precise modes are only based on the simulation in which the physical insight is hard to yield. It is not until recent years that S. Tian [22]-[23] simplified the model from the 5th order to a 3th one with a result of an analytical transfer function for both SRC and LLC converter. Followed by this, Y. Hsieh has employed a novel rotating coordinate modeling concept to the SRC converter and achieved the most precise prediction results based on the describing transfer function [24]-[25], in which the best accurate analytical transfer function has also been extracted but it is still expected to extend to the LLC converter. As a conclusion, there is the challenge for the conventional DFC LLC control to apply to most of the applications due to its not good transient performance by the intrinsic loop characteristic.

To solve this problem, several new LLC loop control schemes have been proposed in recent years. The first one is the average current control [26]-[27], in which the tank current has been sensed and scaled and then participate in the loop control, it is proved to be with the advantages over the DFC control on input ripple cancellation but still owns the room for the further improvement in terms of the load transient performance. The second one is the charge control [28], which compares the total input charge with the control voltage to modulate the switching frequency, then an inner current loop is offered to yield the fast-transient response. Another hysteretic charge control [29]-[30] can also achieve the good dynamic load performance by sensing the current of tank current or voltage of resonant capacitor, but the DC gain of control to output is with a little big variation during the full load range which will bring the difficulty of loop design at light load. However, there are not the detail analytical transfer functions to describe the whole physical insight regarding their small signal characteristics.

In view of the metioned above issues, the HHC control, with the combination of charge current control and hysteretic charge control, is proposed to achieve the best-in-class 
transient performance by overcoming the disadvantage of charge control and avoiding unstable condition by adding into the frequency compensation ramp [31]-[33]. It has changed the plant transfer function to an equivalent first order system characterized by a relatively stable cross frequency and small variation of DC gains, which has made the loop design easy to compensate.

In this paper, the small signal model for new LLC converter will be proposed firstly, which is not purely depend on the approach of extended describing function and the related superposition theorem, but the improved results of a third order transfer function from this theorem. In Section II, the transfer functions of the proposed model are derived and simplified for the purpose of physical insight investigation. Different from the conventional DFC control, the proposed model replaces the small signal variable of $F_{\mathrm{s}}$ by that from feedback control. In Section III and IV, the derived analytical transfer functions regarding the open loop of control to output, input to output, output impedance and that of the closed loop are verified through simulation, in which, the detail physical insight analysis are provided. In section $\mathrm{V}$, the experiment of practical design is conducted to verify the proposed transfer functions. Section VI summarizes the paper.

\section{Small-Signal Model for HHC Control}

As shown in Fig.1, the key difference between HHC and the conventional DFC is the added feedback signal of $\mathrm{VCR}$, which is a combination of the internal current ramp part and the divided scaling part from the voltage of the resonant capacitor $C_{\mathrm{s}}$ [31]-[32]. Refer to Fig. 1, VHS is the input voltage of the half bridge LLC tank, VTL and VTH come from the compensation voltage $V_{\text {comp }}$, they are symmetrical based on a reference voltage VCM (for example $3 \mathrm{~V}$ ) and then will be compared with VCR. So $v_{\text {crl }}$ and $v_{\text {crh }}$ are produced henceforth to provide the input signal for the S/R latch, therefore, the final drive signal Gate_L and Gate_H can be produced based on another deadtime control signal for the $\mathrm{S} / \mathrm{R}$ latch. As shown in Fig. 2, $v_{\text {crl }}$ will turn high and then pull Gate_L low when VTL crosses $\mathrm{VCR}$ at time of $t_{0}$, then the VHS begin to rise, when the rising of VHS completes or the dead time elapses at $t_{1}$, Gate_H will turn high. At the next time of $t_{2}$ when VTH has crossed VCR, $v_{\text {crh }}$ will turn high and then pull Gate $\mathrm{H}$ low, then the VHS begins to decrease, when VHS finishes this process or the dead time has been elapsed at $t_{3}$, Gate_L will turn high.

\section{A. Small Signal Model for LLC HHC Control}

For a conventional DFC control, the simplified analytical transfer functions have been proposed [22]-[23], in which the capacitor small signal model has turned into an inductor small signal model. So, the original small signal model of LLC tank has been simplified greatly from an above fifthorder equivalent circuit into a third-order equivalent circuit. This has provided the great possibility to extend this result

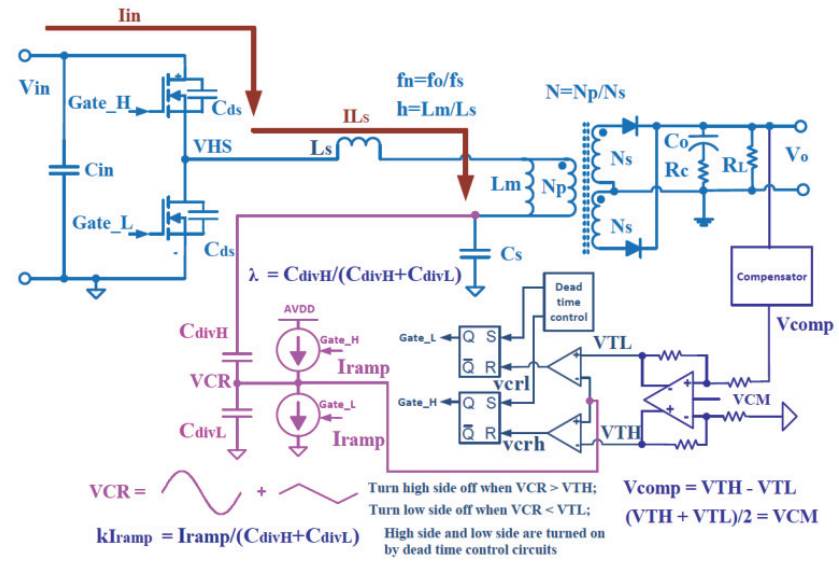

Fig. 1. The proposed HHC control used in LLC HB converter.

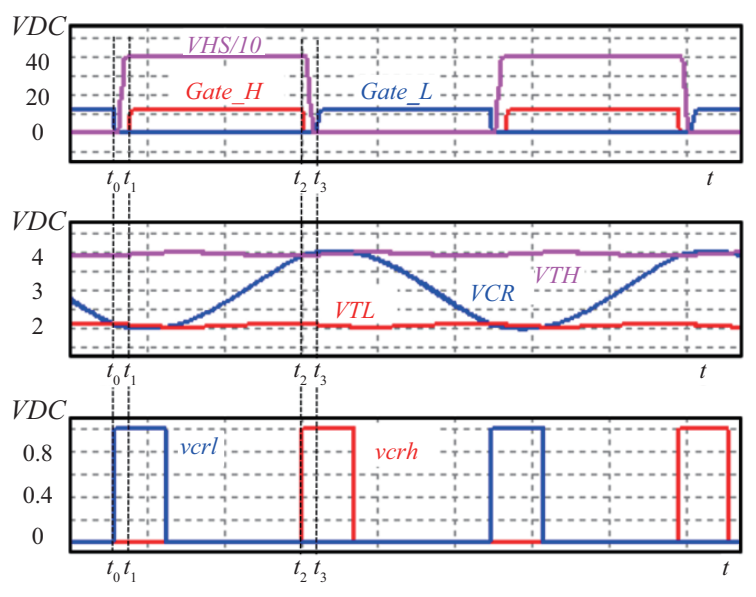

Fig. 2. Proposed hybrid hysteretic control used in LLC converter.

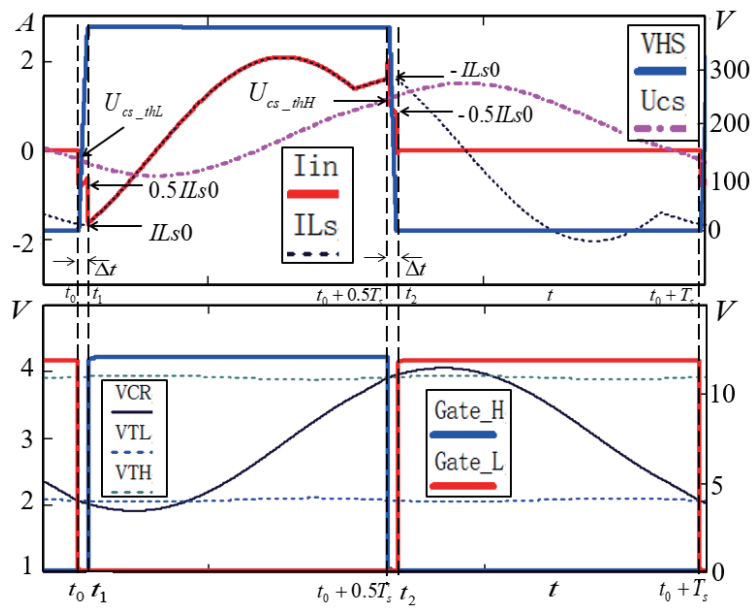

Fig. 3. Waveforms between the tank part and the VCR part for HHC control.

to other kinds of LLC control topology.

As for $\mathrm{HHC}$, it is critical to investigate the key difference compared with DFC, which is the scaling voltage sense of the resonant capacitor voltage. Fig. 3 shows the key waveforms between input current $I_{\text {in }}$, tank current $I_{L \mathrm{~s}}$, voltage of resonant capacitor $U_{\mathrm{cs}}$, tank input voltage VHS, High side Gate drive Gate_H, low side Gate drive Gate_L, VCR, VTH 


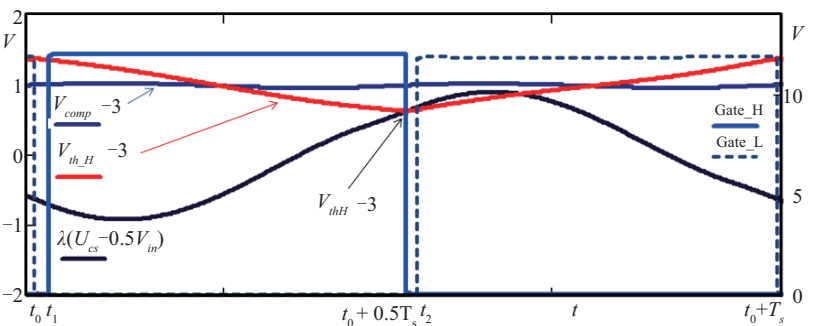

Fig. 4. Operation principle of TI HHC control with ramp current.

and VTL.

As shown in Fig. 1, the scaling voltage part in VCR is obtained from the divided voltage of $C_{\mathrm{s}}$ by the capacitors $C_{\mathrm{divH}}$ and $C_{\text {divL }}$. The scaling down coefficient can be defined as:

$$
\lambda=\frac{C_{\mathrm{divH}}}{C_{\mathrm{divL}}+C_{\text {divH }}}
$$

It is generally assumed the dead time $\Delta t$ of the gate drives to be equal. Fig. 3 shows the details regarding the $I_{\text {in }}$ and VHS, and then the average input current can be obtained as:

$I_{\text {in_avg }}=\frac{\int_{t_{0}}^{t_{0}+0.5 T_{\mathrm{s}}} i_{L S}(t) \mathrm{d} t-0.5 \int_{t_{0}}^{t_{1}} i_{L s}(t) \mathrm{d} t+0.5 \int_{t_{0}+0.5 T_{s}}^{t_{2}} i_{L s}(t) \mathrm{d} t}{T_{\mathrm{s}}}$

If defining the voltage of $U_{\mathrm{cs}}$ at $t_{0}$ and $t_{0}+0.5 T_{\mathrm{s}}$ as $U_{\text {cs thL }}$ and $U_{\text {cs thH }}$, and the tank current as $I_{L s 0}$ at $t_{0}$, then (2) can be re-written as:

$$
\begin{aligned}
I_{\text {in_avg }} & =\frac{\int_{t_{0}}^{t_{0}+0.5 T_{\mathrm{s}}} i_{L s}(t) \mathrm{d} t+2\left|0.5 I_{L s} 0\right| \Delta t}{T_{\mathrm{s}}} \\
& =\frac{\int_{t_{0}}^{t_{0}+0.5 T_{\mathrm{s}}} i_{L s}(t) \mathrm{d} t+2 C_{\mathrm{ds}} V_{\mathrm{in}}}{T_{\mathrm{s}}} \\
& =F_{\mathrm{s}}\left(C_{\mathrm{s}}\left(U_{\text {cs_thH }}-U_{c s \_ \text {thL }}\right)+2 C_{\mathrm{ds}} V_{\text {in }}\right)
\end{aligned}
$$

It shows the minimum dead time is considered from (3), so the input power under steady status can be finally written as:

$$
P_{\text {in }}=V_{\text {in }} F_{\mathrm{s}} C_{\mathrm{s}}\left(2 U_{\text {cs } \_ \text {thH }}-V_{\text {in }}\right)+2 C_{\text {ds }} F_{\mathrm{s}} V_{\text {in }}^{2}
$$

In the configuration of HHC method products such as TI UCC25630X series, the quantity of $U_{\text {cs thH }}$ can be sensed by the voltage of VCR pin even though there is another combination of ramp current by the internal frequency compensation. Shown as in Fig. 4, the scaling voltage $\lambda\left(U_{\mathrm{cs}}{ }^{-}\right.$ $0.5 V_{\text {in }}$ ) will cross th e quantity of $\mathrm{V}_{\text {th }} \mathrm{H}-\mathrm{VCM}$ (the combined quantity of feedback and ramp current) with a value of $V_{\text {thH }}$-VCM when Gate_H turns off, the operation principle will be elaborated by the formulas (5)-(7).

From the operation principle of HHC control, the compensation part in terms of integrating frequency control into VCR voltage is paramount for the investigation, if we define the internal coefficient for the ramp compensation as:

$$
k_{\text {Iramp }}=\frac{I_{\text {ramp }}}{C_{\text {divL }}+C_{\text {divH }}}
$$

Then the following equations can be obtained as:

$$
\begin{gathered}
V_{\text {comp }}-\frac{0.25 k_{\text {Iramp }}}{F_{\mathrm{s}}}=V_{\text {thH }} \\
\lambda\left(U_{\text {cs_thH }}-0.5 V_{\text {in }}\right)+\mathrm{VCM}=V_{\text {thH }}
\end{gathered}
$$

If we define the conversion efficiency from primary side to secondary side as $\eta, V_{\mathrm{f}}$ as the average forward voltage for a rectifier on the output side, $I_{\text {sec }}$ as the LLC tank's output current, $I_{\mathrm{o}}$ as the external load current, and $Z(s)$ as the LLC output impedance, then the following equation can be obtained if the formulas of (5)-(7) are combined into (4).

$I_{\mathrm{sec}}=\frac{\eta\left(V_{\mathrm{in}} F_{\mathrm{s}} C_{\mathrm{s}} \frac{2\left(V_{\mathrm{thH}}-\mathrm{VCM}\right)}{\lambda}+2 C_{\mathrm{ds}} F_{\mathrm{s}} V_{\mathrm{in}}^{2}\right)}{V_{\mathrm{o}}+V_{\mathrm{f}}}=I_{\mathrm{o}}+\frac{V_{\mathrm{o}}}{Z(s)}$

In above (8), there are four variables $V_{\mathrm{o}}, V_{\mathrm{in}}, V_{\mathrm{thH}}$ and $I_{\mathrm{o}}$, which are mainly with DC components but not include the switching frequency components and its harmonics, then the conventional average concept [1] can be applied to derive the related small signal small equation. For the theoretical manipulation, we can ignore the quantities of $V_{\mathrm{f}}$ and $\eta$. Perturbing on the above formula (8) with the quantities of $V_{\mathrm{o}}, V_{\text {in }}, V_{\text {thH }}$ and $I_{\mathrm{o}}$ by $\hat{v}_{\mathrm{o}}, \hat{v}_{\text {in }}, \hat{v}_{\mathrm{thH}}, \hat{i}_{\mathrm{o}}$ respectively, we can obtain the following small signal equations:

$\hat{v}_{\mathrm{o}}=Z(s)\left(-\frac{\hat{v}_{\mathrm{o}}}{R_{L}}+k_{\mathrm{th}} \hat{v}_{\mathrm{thH}}+\left(\frac{V_{\mathrm{o}}}{R_{L} F_{\mathrm{s}}}+\frac{2 C_{\mathrm{ds}} V_{\mathrm{in}}^{2}}{V_{\mathrm{o}}}\right) \hat{f}+k_{\mathrm{in}} \hat{v}_{\mathrm{in}}-\hat{i}_{\mathrm{o}}\right)$,

where:

$Z(s)=\frac{R_{L}\left(1+s R_{c} C_{\mathrm{o}}\right)}{1+s R_{L} C_{\mathrm{o}}} \quad k_{\mathrm{in}}=\frac{V_{\mathrm{o}}}{R_{L} V_{\mathrm{in}}}-\frac{2 C_{\mathrm{ds}} V_{\mathrm{in}} F_{\mathrm{s}}}{V_{\mathrm{o}}} \quad k_{\mathrm{th}}=\frac{2 C_{\mathrm{s}} V_{\mathrm{in}} F_{\mathrm{s}}}{V_{\mathrm{o}} \lambda}$

Refer to Fig. 4, the small signal average concept can also be employed based on (6). Perturbing on the variables of $V_{\text {comp }}, F_{\mathrm{s}}$ and $V_{\text {thH }}$ by $\hat{v}_{\text {comp }}, \hat{f}, \hat{v}_{\text {thH }}$, then we can obtain the following:

$$
\hat{v}_{\text {comp }}+\frac{0.25 k_{\text {Iramp }}}{F_{\mathrm{s}}^{2}} \hat{f}=\hat{v}_{\text {thH }}
$$

Combining (10) into (9), we have:

$$
\begin{aligned}
& \hat{v}_{\mathrm{o}} / Z(s)=k_{\mathrm{v}} \hat{v}_{\mathrm{o}}+k_{\mathrm{th}} \hat{\mathrm{c}}_{\text {comp }}+k_{\mathrm{f}} \hat{f}+k_{\text {in }} \hat{v}_{\text {in }}-\hat{i}_{\mathrm{o}} \\
& k_{\mathrm{f}}=\frac{V_{\mathrm{o}}}{R_{L} F_{\mathrm{s}}}+\frac{2 C_{\mathrm{dd}} V_{\mathrm{in}}^{2}}{V_{\mathrm{o}}}+\frac{0.5 V_{\text {in }} C_{\mathrm{s}} I_{\text {ramp }}}{V_{\mathrm{o}} F_{\mathrm{s}} C_{\text {divH }}}
\end{aligned}
$$




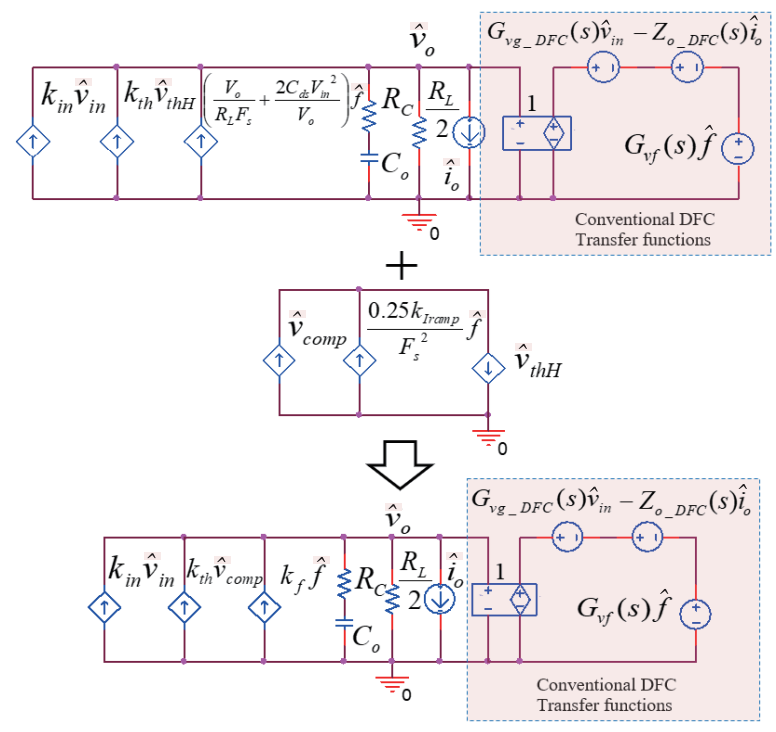

Fig. 5. Small signal equivalent circuit for the LLC HHC control.

So, the correlation in terms of small signal variables among input voltage, output voltage, compensation voltage, operation (7) frequency and external output current have been built by (11).

\section{B. Implementation of the Analytical HHC Small Signal Transfer Function}

Equation (11) shows that the small signal perturbation of the output $V_{\mathrm{o}}$ has one more of the small signal variable $V_{\text {comp }}$ than the conventional DFC control due to the characteristic of HHC control, where the compensation feedback signal is only directly related to Vcomp but not $F_{\mathrm{s}}$ from the control block. It is taken for granted that only one variable $V_{\text {comp }}$ is incorporated into the open loop of control to output finally, then the small signal variable of $F_{\mathrm{s}}$ is required to be cancelled in the final control block. However, it is not straightforward to implement such a behavior in the control block directly; therefore, it is required to make a mathematic manipulation from the conventional DFC control to replace the small signal variable of $F_{\mathrm{s}}$ provided that the analytic small transfer function is given. Refer to the simplified analytic transfer functions applied for a DFC control [23], the perturbation of $V_{\mathrm{o}}$ can be expressed by the open loop transfer functions of input to output $G_{\mathrm{vg} \_ \text {DFC }}(s)$, control to output $G_{\mathrm{vf}}(s)$ and output impendence $Z_{\mathrm{o} \text { DFC }}(s)$, which is shown as:

$$
\hat{v}_{\mathrm{o}}=G_{\mathrm{vg} \_\mathrm{DFC}}(s) \hat{v}_{\mathrm{in}}+G_{\mathrm{vf}}(s) \hat{f}-Z_{\mathrm{o}_{-} \mathrm{DFC}}(s) \hat{i}_{\mathrm{o}}
$$

Fig. 5 shows the small signal equivalent circuit for the LLC HHC control.

Even the concept of extended describing function proposed by Dr. E. Yang has been used widely for the small signal analysis both for SRC, LLC and other types of resonant converters [16], most of the models are accurate enough to make a good prediction regarding the small signal characteristic over a wide frequency range. However, most of them are featured by complicated mathematics expressions or the circuit simulation-oriented way, their analytical based

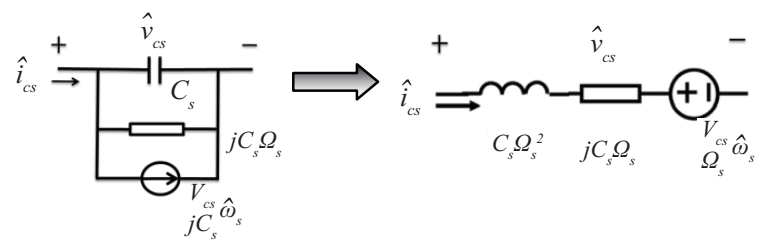

Fig. 6. Simplified equivalent small signal model for the resonant capacitor proposed by S. Tian.

formulas are still hard to be derived due to the higher order of the model. To simplify the order of the model, Shown in Fig. 6. Tian had tried to propose a simplified model successfully by turning the equivalent signal model circuit of the resonant capacitor into an equivalent model featuring inductor behavior [22]-[23], then the original small signal circuit of LLC tank has been simplified greatly from the original fifth-order equivalent circuit into a third-order one, which has provided the great possibility to obtain the analytical expression.

However, the ESR of the output capacitors is neglected due to the practical use of ceramic capacitors as far as the analytical small signal transfer function is concerned [23]. For the scenarios of using electrolytic capacitors, the ESR shall be considered. Shown in Table I, the analytical transfer functions have been modified to achieve this.

The combination of the formulas (11)-(13) will result in a final small signal transfer function shown as below:

$\hat{v}_{\mathrm{o}}(s)\left(\frac{1}{Z(s)}-\left(\frac{k_{\mathrm{f}}}{G_{\mathrm{vf} \_\mathrm{DFC}}(s)}-\frac{1}{R_{L}}\right)\right)$

$=k_{\mathrm{th}} \hat{v}_{\text {comp }}(s)+\left(k_{\mathrm{in}}-\frac{k_{\mathrm{f}} G_{\mathrm{vg} \_\mathrm{DFC}}(s)}{G_{\mathrm{vf} \_\mathrm{DFC}}(s)}\right) \hat{v}_{\mathrm{in}}(s)+\left(\frac{k_{\mathrm{f}} Z_{\mathrm{o}}(s)}{G_{\mathrm{vf} \_\mathrm{DFC}}(s)}-1\right) \hat{i}_{\mathrm{o}}$

Then the transfer function of LLC HHC control can be obtained as follows:

$$
\hat{v}_{\mathrm{o}}=G_{\mathrm{vg} \_ \text {HHC }}(s) \hat{v}_{\mathrm{in}}+G_{\mathrm{vc} \_ \text {HHC }}(s) \hat{v}_{\mathrm{vc}}-Z_{\mathrm{o} \_\mathrm{HHC}}(s) \hat{i}_{\mathrm{o}}
$$

where:

$G_{\mathrm{vc} \_ \text {HHC }}(s)=\frac{k_{\mathrm{th}} Z(s)}{1-Z(s)\left(\frac{k_{\mathrm{f}}}{G_{\mathrm{vf} \_ \text {DFC }}(s)}-\frac{1}{R_{L}}\right)}$

$G_{\mathrm{vg} \_ \text {HHC }}(s)=Z(s)\left(k_{\text {in }}-k_{\mathrm{f}} \frac{G_{\mathrm{vg} \_\mathrm{DFC}}(s)}{G_{\mathrm{vf} \_\mathrm{DFC}}(s)}\right) /\left(1-Z(s)\left(\frac{k_{\mathrm{f}}}{G_{\mathrm{vf} \_\mathrm{DFC}}(s)}-\frac{1}{R_{L}}\right)\right)$

$Z_{\text {o } \_ \text {HHC }}(s)=Z(s)\left(k_{\text {in }}-k_{\mathrm{f}} \frac{Z_{\mathrm{o}_{-} \mathrm{DFC}}(s)}{G_{\mathrm{vf} \_\mathrm{DFC}}(s)}\right) /\left(1-Z(s)\left(\frac{k_{\mathrm{f}}}{G_{\mathrm{v} \mathrm{D}_{-} \mathrm{DFC}}(s)}-\frac{1}{R_{L}}\right)\right)$

If we define $\mathrm{G}_{\mathrm{DC}_{-} \mathrm{vg}}$ as the DC gain of $G_{\mathrm{vg} \_\mathrm{DFC}}(s), \mathrm{G}_{\mathrm{DC}_{-} \mathrm{dm}}$ and $\mathrm{G}_{\mathrm{DC} \mathrm{cm}}$ as the $\mathrm{DC}$ gain of $\mathrm{G}_{\mathrm{vf} D F C}(s)$ under the conditions of $f_{\mathrm{n}} \leqslant 1$ and $f_{\mathrm{n}} \geqslant 1$ respectively ( $f_{\mathrm{n}}$ is the normalized operation frequency), then the transfer functions of open control to output loop $G_{\mathrm{vc} \_ \text {HHC }}(s)$, open input to output loop $G_{\mathrm{vg} \_ \text {HHC }}(s)$, open output impedance $Z_{\mathrm{o} \text { HHC }}(s)$ can be simplified into the analytical formula expression after the mathematic manipulations [33], which is also shown in Table II. 
TABLE I

Transfer Functions for Conventional LLC HB Converter

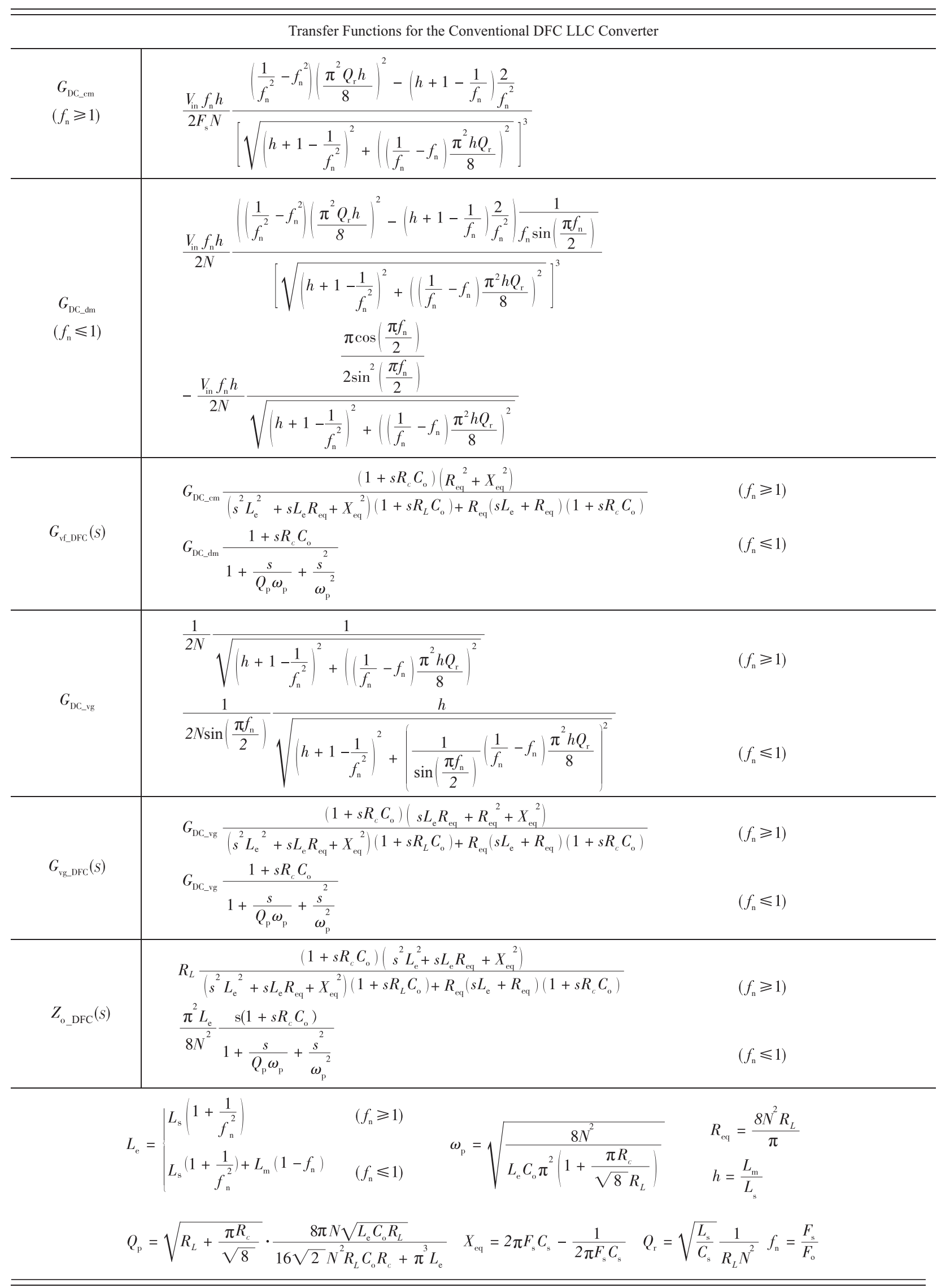


TABLE II

TRANSFER FunCTIONS FOR LLC HHC HB CONVERTER

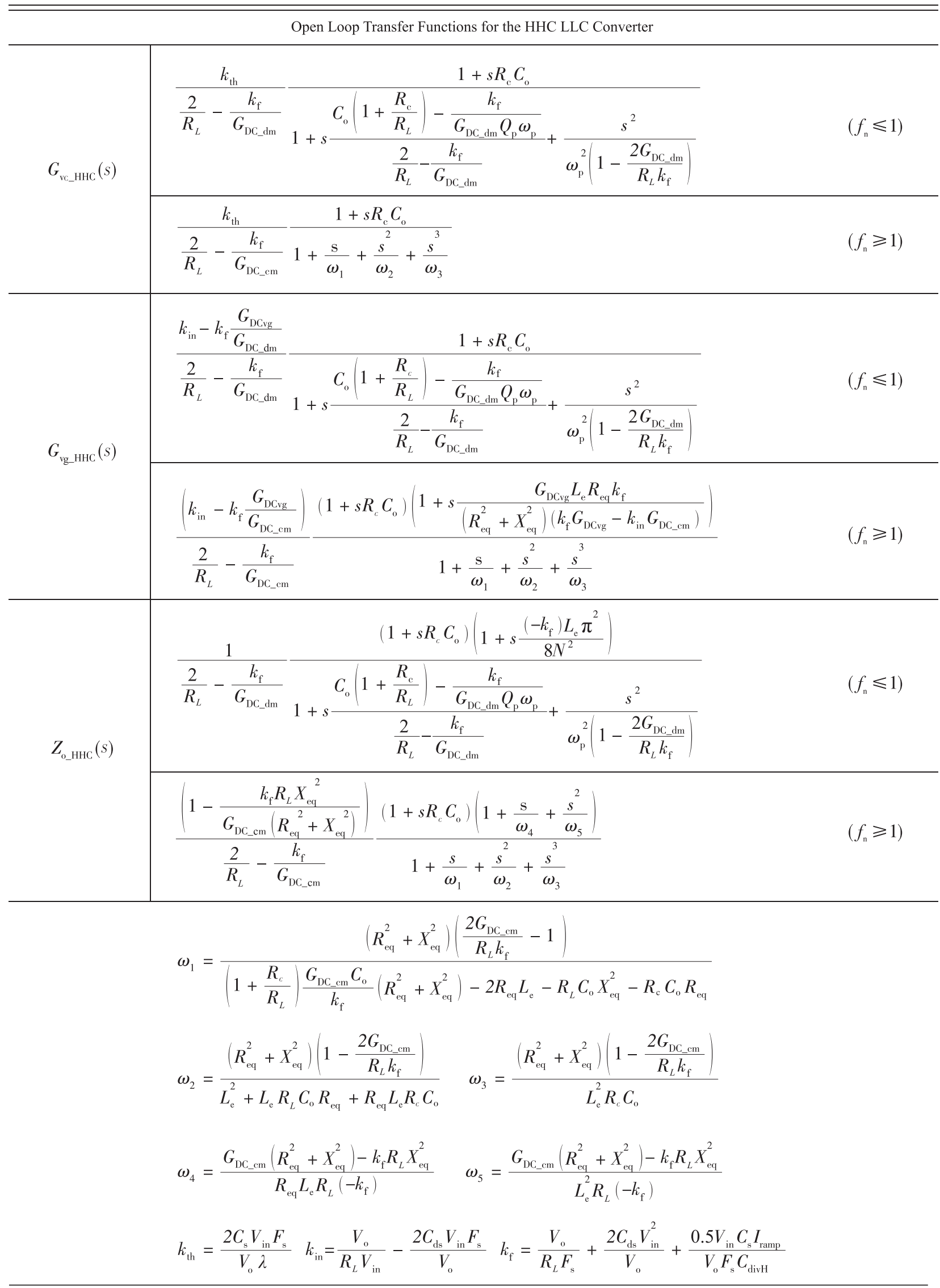




\section{VerificATION ON THE ANALYTICAL TRANSFER FunCNTIONS}

The small signal characteristic of LLC HHC converter will be fully verified and investigated in this section through simulation based on a design example with major power stage parameters setting by: $V_{\text {in }}=370 \mathrm{VDC}-410 \mathrm{VDC}, V_{\mathrm{o}}=$ $24 \mathrm{VDC}, R_{L}=2 \Omega, L_{\mathrm{m}}=330 \mathrm{uH}, L_{\mathrm{s}}=50 \mathrm{uH}, C_{\mathrm{s}}=44 \mathrm{nF}$, $C_{\mathrm{o}}=470 \mathrm{uF} \times 4, R_{c}=24 \mathrm{~m} \Omega / 4, \mathrm{~N}=\mathrm{Np} / \mathrm{Ns}=25 / 3$. The related SIMPLIS simulation is used based on the circuits shown as in Fig. 7.

\section{A. Verification and Analysis on the Transfer Function of Open Control to Output}

Based on the transfer functions presented in Table I and Table II, the open loop transfer functions for the HHC control for the UCC25630X products can be verified further by using the same approach, in which the related small signal plots regarding the open control to output under the scenarios of $f_{\mathrm{n}}>1$ and $f_{\mathrm{n}}<1$ are obtained through calculation. To have a contrast, the simulation is conducted. As shown in Fig. 8, the results between SIMPLIS simulation and calculation are given under the input condition of 370 VDC and 410 VDC with the output being 24 VDC\& $12 \mathrm{~A}$. The dashed lines show the simulation results and the solid lines show the calculation results.

It is shown from Fig. 8 that the calculation results match well with the simulation results in both the conditions. However, the calculation results do not match the simulation results well when frequency increases up to half of the operation frequency due to the simplified third-order of the model and the incorporated average concept, but it does not have any impact on the practical prediction.

In order to investigate the characteristic of $\mathrm{HHC}$ and the advantage over the conventional hysteretic control, the DC gain of $G_{\mathrm{vc}}(s)$ for these two approaches are analyzed. Refer to Table I, the DC gain of $G_{\mathrm{vc}}(s)$ can be simplified as:

$$
\begin{aligned}
& G d c_{\mathrm{vc} \_ \text {HHC }}= \frac{\frac{2 C_{\mathrm{s}} V_{\mathrm{in}} F_{\mathrm{s}}}{V_{\mathrm{o}} \lambda}}{\frac{2}{R_{L}}-\left(\frac{V_{\mathrm{o}}}{R_{L}}+\frac{V_{\mathrm{in}}}{V_{\mathrm{o}}} k_{\text {Iramp }}\right) \frac{1}{G_{\mathrm{DC} \_\mathrm{vf}} F_{\mathrm{o}} f_{\mathrm{n}}}} \\
&=\frac{\frac{4 N C_{\mathrm{s}} F_{\mathrm{o}} f_{\mathrm{n}}}{G_{\mathrm{DC} \_\mathrm{vg}} \lambda}}{\frac{2}{R_{L}}+\left(\frac{V_{\mathrm{o}}}{R_{L}}+\frac{2 k_{\text {Iramp }} N}{G_{\mathrm{DC}_{-} \mathrm{vg}}}\right) \frac{1}{\left(-G_{\mathrm{DC} \_\mathrm{vf}}\right) F_{\mathrm{o}} f_{\mathrm{n}}}}
\end{aligned}
$$

While

$$
G_{\mathrm{DC} \_ \text {vf }}=\left\{\begin{array}{ll}
G_{\mathrm{DC} \_\mathrm{cm}} & (f \geqslant 1) \\
G_{\mathrm{DC} \_\mathrm{dm}} & (f \leqslant 1)
\end{array} k_{\mathrm{n}} \leqslant 1 \text { Iramp }=\frac{C_{\mathrm{s}} \cdot 10^{-3}}{C_{\mathrm{divH}}}\right.
$$

For the HHC, the DC Gain will be inversely proportional to $\lambda$ from (15), another variable is $k_{\text {Iramp }}$, in which the variable

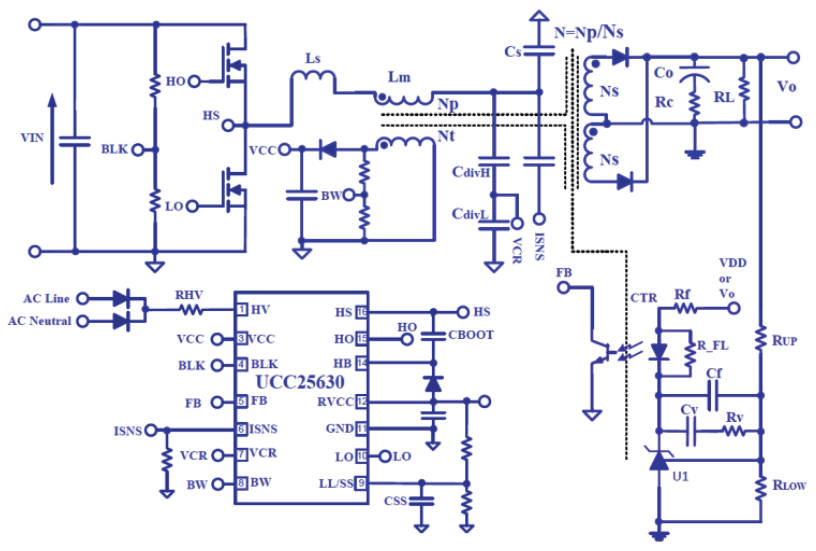

Fig. 7. Circuit for the simulation verification (dead time is set as $200 \mathrm{~ns}$ ).

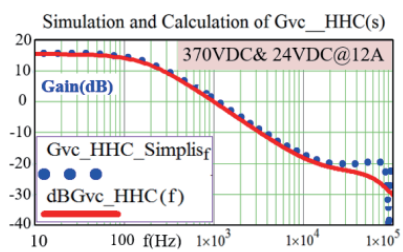

Simulation and Calculation of Gvc_HHC(s)

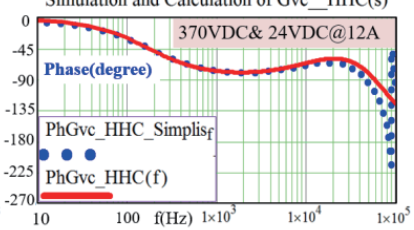

Simulation and Calculation of Gvc_HHC(s)

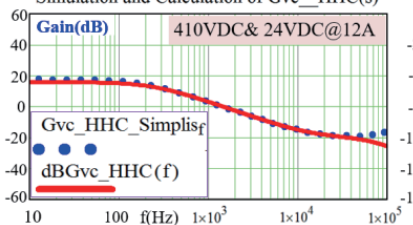

Simulation and Calculation of Gve_HHC(s)

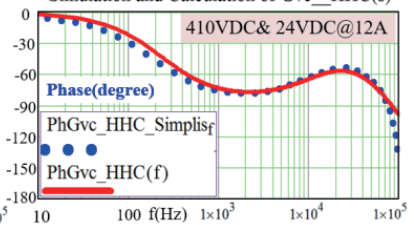

Fig. 8. Plot comparison of control to output between simulation and calculation under the condition of 370 VDC@24 VDC\&12 A $\left(f_{\mathrm{n}}=0.82\right)$ and 410 VDC $\left(f_{\mathrm{n}}=1.08\right) @ 24$ VDC\&12 A.

of $I_{\text {ramp }}$ has been incorporated. However, it is not the case for the conventional charge control. So, the difference can be found through the approximate DC gain plot referring to the variables of $\mathrm{R}_{L}$ and $f_{\mathrm{n}}$. Shown in (16), the normal constant of $k_{\text {Iramp }}$ can be removed and the DC gain for the conventional charge control can be obtained.

$$
G d c_{\mathrm{vc} \_ \text {HHC }}\left(R_{L}, f_{\mathrm{n}}\right)=\frac{\frac{4 N C_{\mathrm{s}} F_{\mathrm{o}} f_{\mathrm{n}}}{G_{\mathrm{DC} \_ \text {vg }}\left(R_{L}, f_{\mathrm{n}}\right) \lambda}}{\frac{2}{R_{L}}+\left(\frac{V_{\mathrm{o}}}{R_{L}}+\frac{2 k_{\text {Iramp }} N}{G_{\mathrm{DC} \_ \text {vg }}\left(R_{L}, f_{\mathrm{n}}\right)}\right) \frac{1}{\left(-G_{\mathrm{DC} \_\mathrm{vf}}\left(R_{L}, f_{\mathrm{n}}\right)\right) F_{\mathrm{o}} f_{\mathrm{n}}}}
$$

With the incorporation of $G_{\mathrm{DC} \text { vg }}\left(R_{L}, f_{\mathrm{n}}\right)$ and $G_{\mathrm{DC} \text { vf }}\left(R_{L}, f_{\mathrm{n}}\right)$ shown in Table I, the approximate DC gain plot between the $\mathrm{HHC}$ control and the conventional hysteretic charge (HC) control can be illustrated in Fig. 9. To have the investigation on the DC gain over a practical design input voltage range, the plotting under different output load for HHC control is shown in the left part of Fig. 9, where three items of the curve tendency can be observed: firstly, the overall DC gain is proportional to $V_{\text {in }}$ under a certain output load even though there is a special case when $f_{\mathrm{n}}$ is below 1 , in which the gain will not decrease at once but it will decrease finally with respect to the decreasing of $f_{\mathrm{n}}$. Secondly, the 


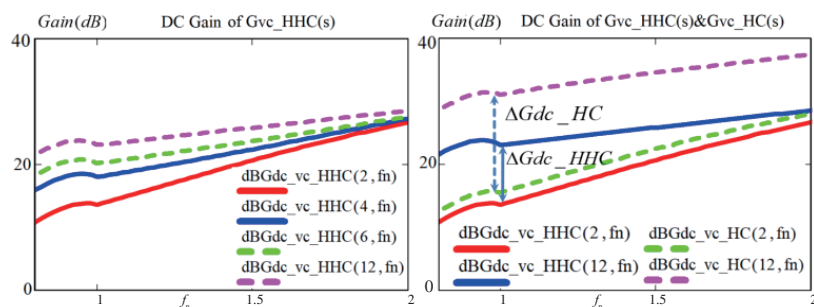

Fig. 9. DC gain plot comparison between the HHC and the conventional hysteretic charge control.

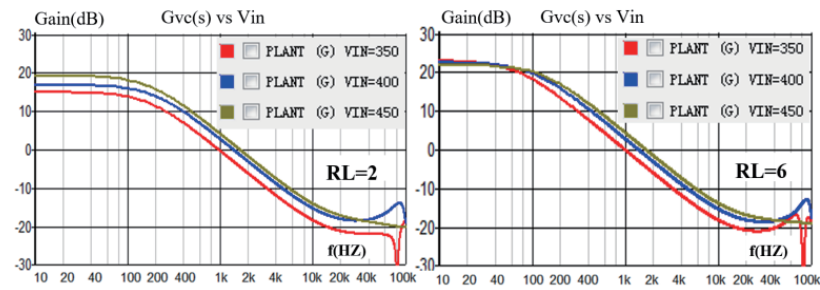

Fig. 10. Loop simulation of control to output when the input voltage varies.

gain will decrease when more of the output current is loaded, which means it will increase under a light load condition. To the third, the delta value of DC gain under those two different load conditions will decrease when $f_{\mathrm{n}}$ is increased. As shown in the right part of Fig. 9, we can obtain the comparison results between proposed HHC control and conventional hysteric charge control: firstly, it is concluded that the gain in $\mathrm{HC}$ control is with the similar operation discipline, it will increase when $f_{\mathrm{n}}$ increases under a certain output current, but the delta value of DC gain under those two conditions is different. Refer to right part of Fig. 9, the solid line and the dashed line represent the plot curves of $\mathrm{HHC}$ control and $\mathrm{HC}$ control respectively, it shows the DC gain of $\mathrm{HC}$ control nearly approaches that of HHC control under full load condition, but it increases more under light load condition. As a conclusion, the control design for a conventional hysteric charge control still faces some challenges if compared to $\mathrm{HHC}$ control.

To verify this conclusion, the simulation is conducted under two different output load conditions of $R_{L}=2 \Omega$ and $6 \Omega$ when input voltage is varied from $350 \mathrm{VDC}$ to $450 \mathrm{VDC}$. Shown as in Fig. 10, it can be observed that the DC gain follows the similar operation discipline concluded above: the gain will decrease as output load increases from $R_{L}=6 \Omega$ to $R_{L}=2 \Omega$, the delta value of the gain under a same varied input range will decrease as output load decreases from $R_{L}=2 \Omega$ to $R_{L}=6 \Omega$.

Fig. 11 shows another investigation when the output varies under a certain input, it can be concluded that the DC gain of the $\mathrm{Gvc}(\mathrm{s})$ has some small variation under the rated output load, the lower the input voltage is, the lower the gain can be obtained. From another point of review, the DC gain will decrease with the increase of output load under these two input conditions, the delta value of the gain under a same varied output load range will decrease with the increase of input voltage.

It shows from Figs.10-11 that the cross frequency for the closed loop shall be below the zero of output electrolytic capacitors and also required to avoid the impact range of high frequency dynamics occurring behavior. In order

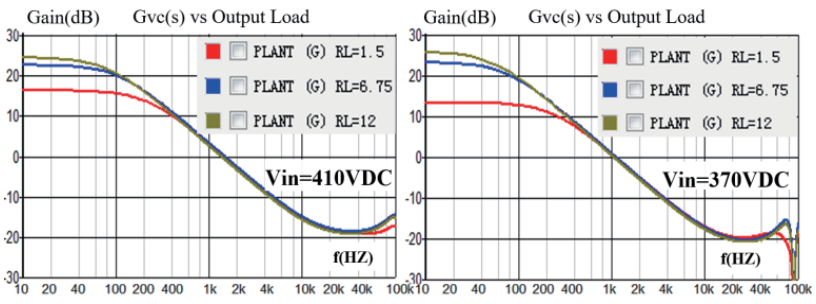

Fig. 11. Sweep of control to output when output current varies.

to yield an easy design for the compensation circuit, the transfer function of open control to output is required to be simplified. Take the scenario of $f_{\mathrm{n}} \geqslant 1$, for example, if the high order poles shown in $G_{\mathrm{vc}}(s)$ are neglected, then the transfer function of open control to output can be simplified further as follows:

$$
G_{\mathrm{vc} \_ \text {HHC_design }}(s) \approx \frac{k_{\mathrm{th}}}{\frac{2}{R_{L}}-\frac{k_{\mathrm{f}}}{G_{\mathrm{DC} \_\mathrm{cm}}}} \frac{1+s R_{c} C_{\mathrm{o}}}{1+\frac{s}{\omega_{1}}} \quad\left(f_{\mathrm{n}} \geqslant 1\right)
$$

The cross frequency can be approximated as:

$f_{\mathrm{c} \_ \text {Gvc }}=\frac{\omega_{1}}{2 \pi} \frac{k_{\mathrm{th}}}{\frac{2}{R_{L}}-\frac{k_{\mathrm{f}}}{G_{\text {DC_cm }}}}=\frac{1}{2 \pi} \frac{k_{\mathrm{th}} / k_{\mathrm{f}}}{\frac{C_{\mathrm{o}}}{k_{\mathrm{f}}}-\frac{2 R_{\text {eq }} L_{\mathrm{e}}+R_{L} C_{\mathrm{o}} X_{\text {eq }}^{2}}{G_{\text {DC_cm }}\left(X_{\text {eq }}{ }^{2}+R_{\text {eq }}{ }^{2}\right)}}$

The above (18) can be further simplified as:

$$
\begin{gathered}
f_{\mathrm{c} \_ \text {Gvc }}=\frac{1}{2 \pi} \frac{\frac{k_{\mathrm{th}}}{k_{\mathrm{f}}}}{\frac{C_{\mathrm{o}}}{k_{\mathrm{f}}}-\delta\left(R_{L}, f_{\mathrm{n}}\right)} \\
\delta\left(R_{L}, f_{\mathrm{n}}\right)=\frac{R_{L} L_{\mathrm{s}} f_{\mathrm{n}} h\left(\frac{16 N^{2}}{\pi^{2}}+\frac{C_{\mathrm{o}}}{C_{\mathrm{s}}}\left(f_{\mathrm{n}}-\frac{1}{f_{\mathrm{n}}}\right)^{2}\right)}{2 V_{\mathrm{o}}\left(\frac{L_{\mathrm{s}}}{C_{\mathrm{s}}}\left(f_{\mathrm{n}}-\frac{1}{f_{\mathrm{n}}}\right)^{2}+R_{L}^{2}\left(\frac{8 N^{2}}{\pi^{2}}\right)^{2}\right)}
\end{gathered}
$$

The investigation regarding the cross frequency on (19) has been revealed that the factor $\delta\left(R_{\mathrm{L}}, f_{\mathrm{n}}\right)$ is much smaller than the value of the variable $C_{0} / k_{\mathrm{f}}$ in the practical application, then the cross frequency can be simplified further as:

$$
f_{\mathrm{c} \_ \text {Gve }} \approx \frac{1}{2 \pi} \frac{k_{\mathrm{th}}}{C_{\mathrm{o}}}=\frac{C_{\mathrm{s}} V_{\mathrm{in}} F_{\mathrm{s}}}{\pi \lambda V_{\mathrm{o}} C_{\mathrm{o}}}
$$

Equation (20) shows that the cross frequency for the open control to output is firmly related to the operation frequency no matter whatever the output load is, which can be verified from the results shown in Fig. 11, where the cross frequency is proved not to be affected by the output load directly. 


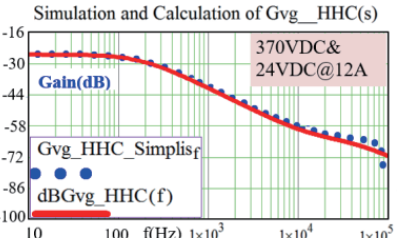

Simulation and Calculation of Gvg $\mathrm{HHC}(\mathrm{s})$

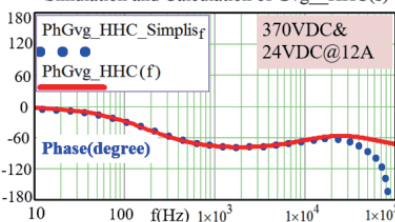

Simulation and Calculation of Gvg_HHC(s)

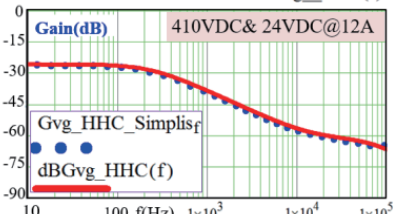

Simulation and Calculation of Gvg HHC(s)

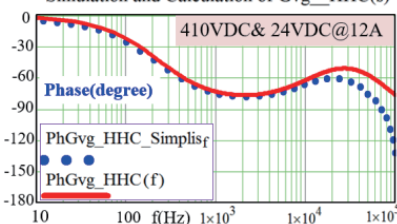

Fig. 12. Plot comparison of input to output between simulation and calculation under the condition of 370 VDC@24 VDC\&12 A $\left(f_{\mathrm{n}}=0.82\right)$ and 410 VDC $\left(f_{\mathrm{n}}=1.08\right) @ 24 \mathrm{VDC} \& 12 \mathrm{~A}$.

Another scenario of $f_{\mathrm{n}} \leqslant 1$ can also be verified by using the same approach mentioned above. With this characteristic, it is concluded that the compensation circuit can be easily designed for the HHC control.

\section{B. Verification and Analysis on the Analytical Transfer Function of Audio Susceptibility}

To investigate the audio susceptibility, the plot of open transfer function of the input to output is carried out. Fig. 12 illustrates such a result comparison between simulation and calculation in both the input condition of $370 \mathrm{VDC}$ and $410 \mathrm{VDC}$. It is revealed that the mathematical calculation results match well with the simulation results except the occurrence of the error when the frequency goes up to half of the operation frequency.

As shown in Fig. 12, it can be seen the gain decreases more after the first poles $\omega_{T}\left(f_{\mathrm{n}} \leqslant 1\right)$ and $\omega_{1}\left(f_{\mathrm{n}} \geqslant 1\right)$. As for the practical applications where the ripple frequency of PFC is around $100 \mathrm{~Hz}$, the closed audio acceptability shall be firmly related to the DC gain of the transfer function. Equation (21) shows the formulas of the DC gain based on the variables of output load and the normalized frequency, the approximated gain can be obtained by (22) at the operation condition of the resonant frequency. As for the conventional HC control, the DC gain can be obtained by setting $k_{\text {ramp }}$ as zero based on (21)-(22).

$$
\begin{aligned}
& G_{\mathrm{DC} \_ \text {vg_HHC }}\left(R_{L}, f_{\mathrm{n}}\right)= \\
& \frac{\frac{G_{\mathrm{DC} \_ \text {vg }}\left(R_{L}, f_{\mathrm{n}}\right)}{2 N \cdot R_{L}}-\left(\frac{V_{\mathrm{o}}}{R_{L}}+\frac{2 k_{\text {Iramp }} N}{G_{\mathrm{DC} \_ \text {vg }}\left(R_{L}, f_{\mathrm{n}}\right)}\right) \cdot \frac{G_{\mathrm{DC} \_\mathrm{vg}}\left(R_{L}, f_{\mathrm{n}}\right)}{2 f_{\mathrm{n}} F_{\mathrm{o}} N G_{\mathrm{DC} \_ \text {vf }}\left(R_{L}, f_{\mathrm{n}}\right)}}{\frac{2}{R_{L}}+\left(\frac{V_{\mathrm{o}}}{R_{L}}+\frac{2 k_{\text {Iramp }} N}{G_{\mathrm{DC}_{-} \mathrm{vg}}\left(R_{L}, f_{\mathrm{n}}\right)}\right) \frac{1}{\left(-G_{\mathrm{DC}_{-} \mathrm{vf}}\left(R_{L}, f_{\mathrm{n}}\right)\right) f_{\mathrm{n}} F_{\mathrm{o}}}}
\end{aligned}
$$$$
D C_{\text {_Gvg_HHC_fo }} \approx \frac{V_{\mathrm{o}}+\left(V_{\mathrm{o}}+\frac{V_{\text {in }} k_{\text {Iramp }}}{I_{\mathrm{o}}}\right) \frac{h}{2}}{2 V_{\text {in }}+N\left(V_{\mathrm{o}}+\frac{V_{\text {in }} k_{\text {Iramp }}}{I_{\mathrm{o}}}\right) h}
$$

Fig. 13 illustrates the operation principle regarding the DC
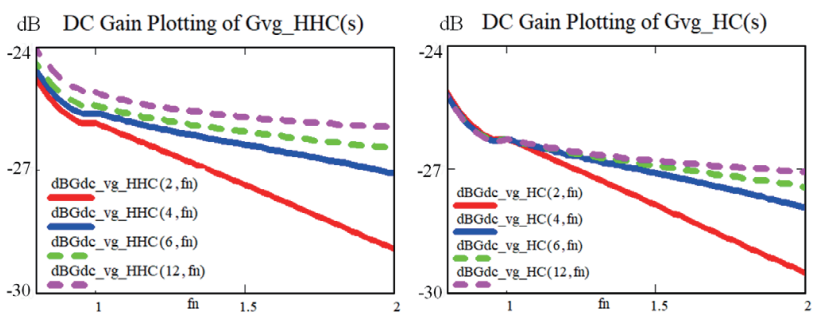

Fig. 13. DC gain plot of input to output under HHC control and conventional HC control.
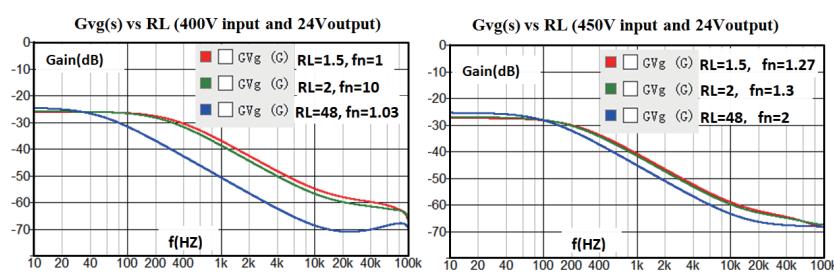

Fig. 14. Plot of input to output under the variation of output load.

gain versus output load for both the conditions under HHC control and $\mathrm{HC}$ control. It is revealed that the gain decreases with the increase of $V_{\text {in }}$ or output current for HHC control. As shown in the left part of Fig. 13, the gain is with small variation when LLC operators near the resonant frequency $\left(f_{\mathrm{n}}=1\right)$ during the load range of $2 \mathrm{~A}$ to $12 \mathrm{~A}$. However, it drops much as the output current increases. As a contrast, the gain nearly remains the same when LLC operates below the resonant frequency point $\left(f_{\mathrm{n}}<1\right)$ for the conventional $\mathrm{HC}$ control and the overall variation shall be relatively smaller than that in the HHC control.

To verify this principle for HHC control, the simulation is implemented to make load sweep analysis based on the transfer function of $G_{\mathrm{vg} \_\mathrm{HHC}}(s)$ when input is $400 \mathrm{VDC}$ and 450 VDC respectively. As shown in Fig. 14. the DC gain increases as output current decreases for both conditions, which matches the result obtained from Fig. 13, the overall variation is small when LLC operates at the resonant frequency point and the gain at $450 \mathrm{VDC}$ is relatively smaller than that of the condition at $400 \mathrm{VDC}$, which is consistence with the calculation result in (21). With this analysis, we can refer to (22) for the further physical insight analysis regarding the practical design as far as the closed transfer function is concerned.

\section{Close the Voltage Loop}

Based on the good match between theoretical calculation and simulation for the open loop control-to-output transfer function, the feedback voltage loop can be constructed for the overall closed loop stabilization. Fig. 15 shows two kinds of compensation circuit regarding the way of $R_{f}$ 's power connection, one way is connecting to the output $V_{\mathrm{o}}$, another way is connecting to a regulated voltage VDD. In this paper, let's take the second connection way for example, in which only the DC gain of the OPTO is considered to have a simple transfer function derivation for the compensation circuit. 


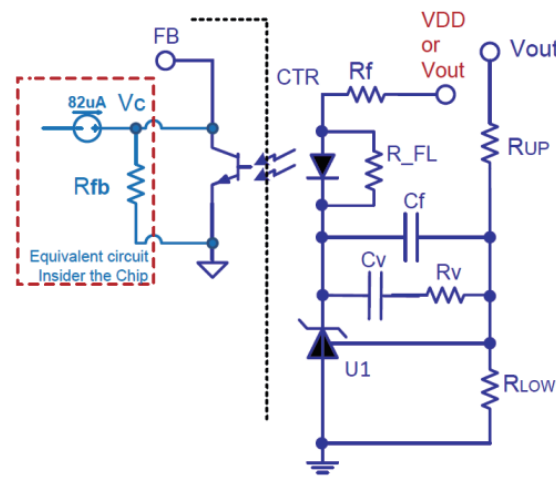

Fig. 15. Feedback circuit for the loop compensation.
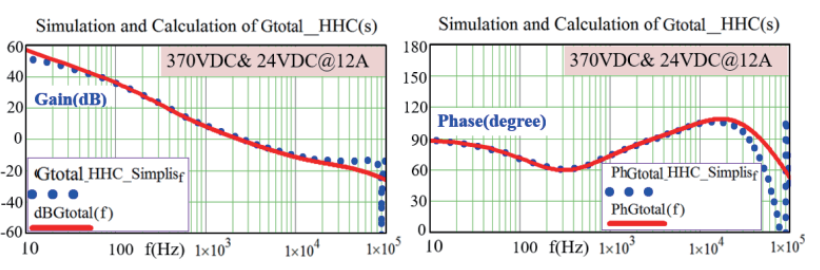

Simulation and Calculation of Gtotal_HHC(s)

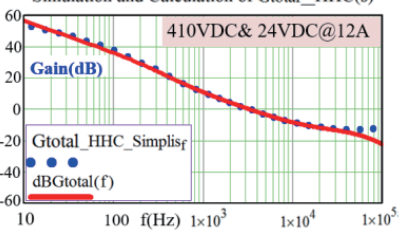

Simulation and Calculation of Gtotal_HHC(s)

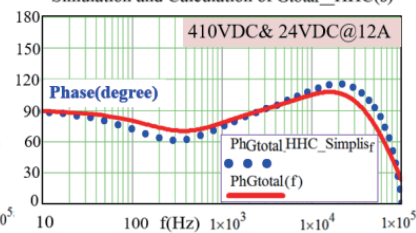

Fig. 16. Overall loop plot comparison between simulation and calculation under the condition of 370 VDC@24 VDC\&12 A $\left(f_{\mathrm{n}}=0.82\right)$ and 410 VDC $\left(f_{\mathrm{n}}=1.08\right) @ 24 \mathrm{VDC} \& 12 \mathrm{~A}$.

Shown in Fig. 15, the parameters are set by $R_{\text {up }}=97.7 \mathrm{k} \Omega$, $R_{\mathrm{v}}=60 \mathrm{k} \Omega, C_{\mathrm{v}}=4.7 \mathrm{nF}, R_{\mathrm{f}}=5 \mathrm{k} \Omega, C_{\mathrm{f}}=20 \mathrm{pF}$, and CTR $=0.35$. Care need to be taken that the additional soft ramp circuit for the purpose of avoiding the output overshot may be suggested to add in the practical design. As for the UCC25630x series, it is specially noted that the equivalent circuit consist of $82 \mathrm{uA}$ inner current source and $R_{\mathrm{fb}}$ insider the chip shall be considered to form a whole compensation circuit. Since $V_{\text {comp }}$ in $G_{\text {vс ннС }}(s)$ is 0.5 times of the voltage on $R_{\mathrm{fb}}$ inside the chip from Table II, we can use this value for the calculation. So, the transfer function for the compensation circuit can be obtained as:

$$
G_{\mathrm{cv}}(s)=\frac{R_{\mathrm{fb}} \mathrm{CTR}}{R_{\mathrm{f}}} \frac{1}{\left(C_{\mathrm{v}}+C_{\mathrm{f}}\right) \cdot R_{\text {up }}} \frac{1+R_{\mathrm{v}} C_{\mathrm{v}} s}{\left(1+\frac{s \cdot R_{\mathrm{v}} C_{\mathrm{v}} C_{\mathrm{f}}}{C_{\mathrm{v}}+C_{\mathrm{f}}}\right) s}
$$

The overall loop is then obtained as follows:

$$
G_{\text {total }}(s)=G_{\text {vс_HнС }}(s) G_{\text {cv }}(s)
$$

Fig. 16 shows the plot comparison of the overall loop between simulation and calculation under the input condition of $370 \mathrm{VDC}$ and $410 \mathrm{DC}$ respectively.

It shows the good matched result between the simulation and calculation can be achieved within a wide range of
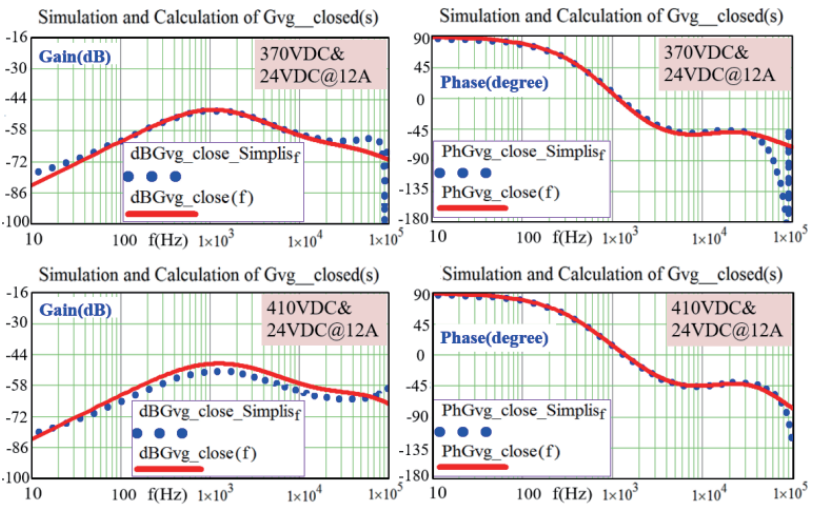

Fig. 17. Results comparison of closed audio susceptibility between simulation and calculation under the condition of 370 VDC@24 VDC\&12 A $\left(f_{\mathrm{n}}=0.82\right)$ and 410 VDC $\left(f_{\mathrm{n}}=1.08\right) @ 24$ VDC\&12 A.

frequency, in which both the cross frequency and phase margin are nearly the same. The cross frequency of the closed loop is nearly in proportion to the operation frequency in these two conditions.

\section{Characteristics Under Line Change And LoAd Change}

\section{A. The Closed Audio Susceptibility and Characteristic of AC Ripple Rejection}

Based on $G_{\text {vg_Hнс }}(s)$ shown in Table I, the closed audio susceptibility can be obtained as follows:

$$
G_{\mathrm{vg} \_ \text {closed }}(s)=\frac{G_{\mathrm{vg} \_ \text {HHC }}(s)}{1+G_{\mathrm{vc} \_ \text {HHC }}(s) \cdot G_{\mathrm{cv}}(s)}
$$

To verify the result at the condition of 12 A output, the comparison between simulation and calculation under $370 \mathrm{VDC}$ and 410 VDC can be obtained as shown in Fig. 17.

It can be seen the good matched results can be achieved between the simulation and calculation and then it can be proposed to evaluate converter's line regulation by applying into the time domain.

To characterize the input voltage rejection, the PFC input voltage is applied by a combinated value of DC part and AC ripple part. Based on the $\mathrm{AC}$ line frequency of $F_{\text {line }}$, the $\mathrm{AC}$ voltage part can be written as:

$$
V_{\text {in_AC }}(t)=V_{\text {PFC_AC }} \sin \left(2 \pi F_{\text {line }} t\right)
$$

So, the corresponding output voltage can be calculated as:

$$
V_{\text {o_in }}(t)=V_{\text {o_ } \_ \text {DC }}+V_{\text {in_AC }}(t) G_{\text {vg_closed }}\left(j \cdot 2 \pi F_{\text {line }}\right)
$$

Take the condition of $f_{\mathrm{n}}>1$ for example, when the input varies from $405 \mathrm{~V}$ to $425 \mathrm{~V}$ based on an $\mathrm{AC}$ line frequency of $100 \mathrm{~Hz}$, the calculation result of output from (27) is shown in the right part of Fig. 18, it reveals the average variation of 

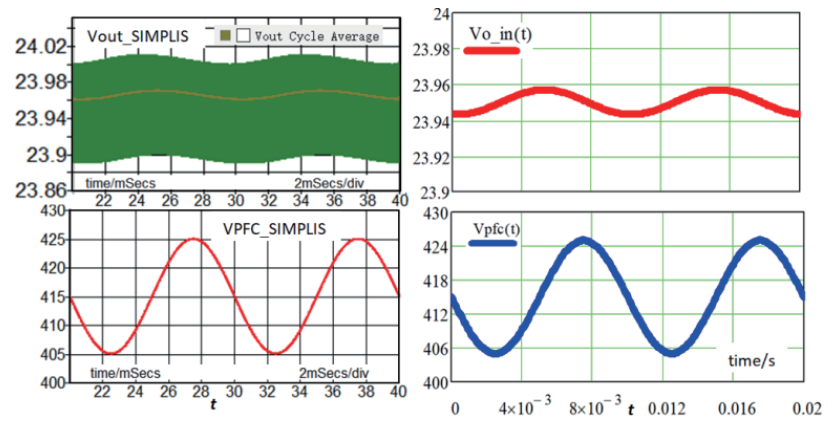

Fig. 18. Comparison of the output between calculation and simulation, in which PFC is with a 20 VAC ripple based on the input of $415 \mathrm{VDC}$.
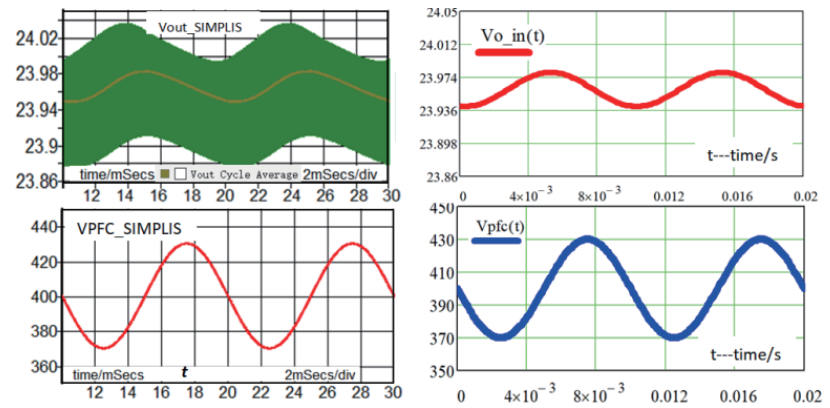

Fig. 19. Comparison of output between calculation and simulation when PFC is with $60 \mathrm{~V}$ 's AC ripple based on 400 VDC input.

output ripple is only $20 \mathrm{mVDC}$, which is consistent with the result of simulation shown in the left part of Fig. 18.

To demonstrate the input ripple rejection characteristic further, a big ripple of $60 \mathrm{VAC}$ is applied based on the $400 \mathrm{VDC}$ under the same line frequency, the comparison results between simulation and calculation are obtained as shown in Fig. 19, which is observed that the output variation from the calculation is $38 \mathrm{mV}$, which matches well with the simulation result.

So, it is concluded that the LLC HHC control has the advantage over the conventional DFC control regarding the input voltage rejection, which means the decreased value of PFC electrolytic capacitors for $\mathrm{HHC}$ control will not affect the performance of output characteristic.

\section{B. The Closed Output Impedance and Dynamic Load Transient Characteristic}

Since the transfer function of open output impedance is derived in Table II, it can be used to obtain the closed output impedance, which is shown as:

$$
Z_{\text {out_closed }}(s)=\left.\frac{\Delta v_{\mathrm{o}}}{\Delta i_{\mathrm{o}}}\right|_{\text {closed }}=\frac{-Z_{\mathrm{o} \_ \text {HHC }}(s)}{1+G_{\mathrm{vc} \_ \text {HHC }}(s) G_{\mathrm{cv}}(s)}
$$

The comparison between simulation and calculation under the input condition of $370 \mathrm{VDC}$ and $410 \mathrm{VDC}$ and the output condition of 24 VDC\&12 A can be obtained from Fig. 20. It shows the results match well over a wide frequency range,
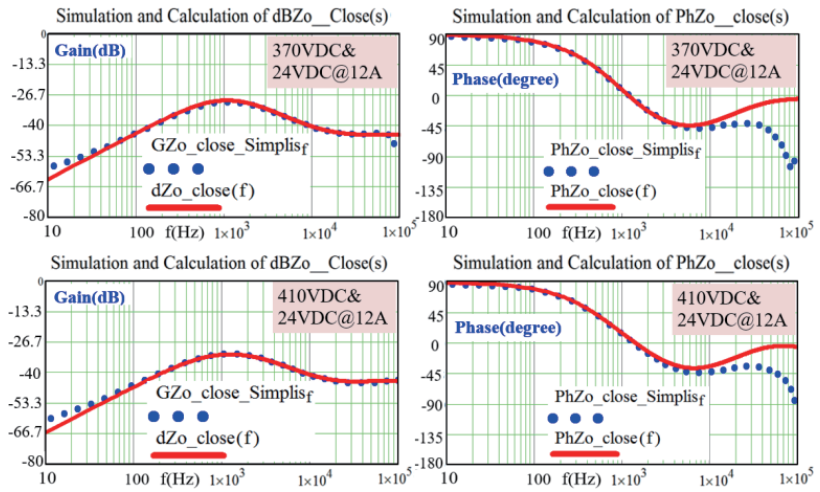

Fig. 20. The plot comparison of closed output impendence between simulation and calculation under the condition of 370 VDC@24 VDC\&12 A $\left(f_{\mathrm{n}}=0.82\right)$ and 410 VDC $\left(f_{\mathrm{n}}=1.08\right) @ 24 \mathrm{VDC} \& 12 \mathrm{~A}$.
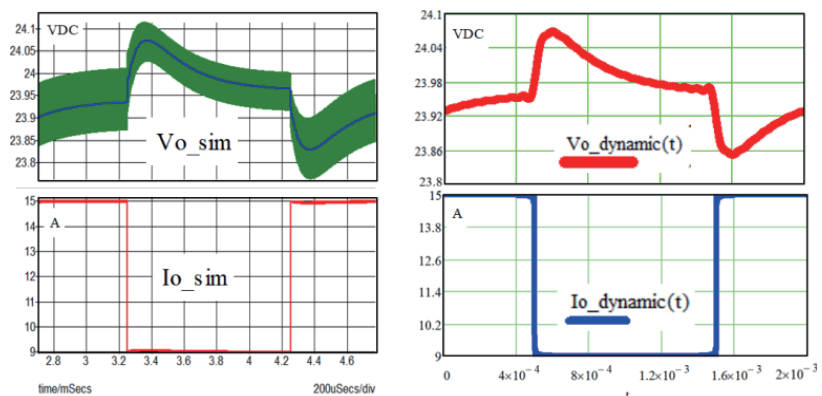

Fig. 21. Results comparison between simulation and calculation under the condition of 410 VDC input and 24 VDC\&9 A-15 A-9 A output.

especially for the bode plot. However, there is some variation for the phase plot comparison when frequency approaches the operation frequency. Further investigation shows it is caused by the non-precise zeros of $Z_{\mathrm{o} \mathrm{HHC}}(s)$ occurred in the high frequency range due to the averaged concept employing on a simplified LLC tank model [22]-[23], which has been hard for us to obtain the precise high frequency dynamics. However, the phase calculation will match the simulation well if the high frequency zeros are assumed high beyond the operation frequency. Then the closed output load dynamic response can be investigated through the conversion from frequency domain into time domain based on (28).

Since the dynamic step load can be simplified by a squared wave with a certain repetition frequency $f_{t s}$, the equation for the square wave under the transient frequency $f_{t s}$ can be expressed as a Fourier series, which is given by:

$$
i_{\text {o_dynamic }}(t)=\frac{2 I_{\text {step }}}{\pi} \cdot \sum_{n=1}^{\infty} \frac{(-1)^{n=1}}{2 n-1} \cos \left((2 n-1) \cdot 2 \pi f_{t s} t\right)
$$

where $I_{\text {step }}$ is the load step and the corresponding output voltage can be calculated as:

$$
V_{\text {o_dynamic }}(t)=V_{\text {o }}+i_{\text {o_dynamic }}(t) Z_{\text {out_closed }}\left(j \cdot 2 \pi f_{t s}\right)
$$

Fig. 21 shows the comparison results between simulation and calculation under the input condition of $410 \mathrm{VDC}$ and 


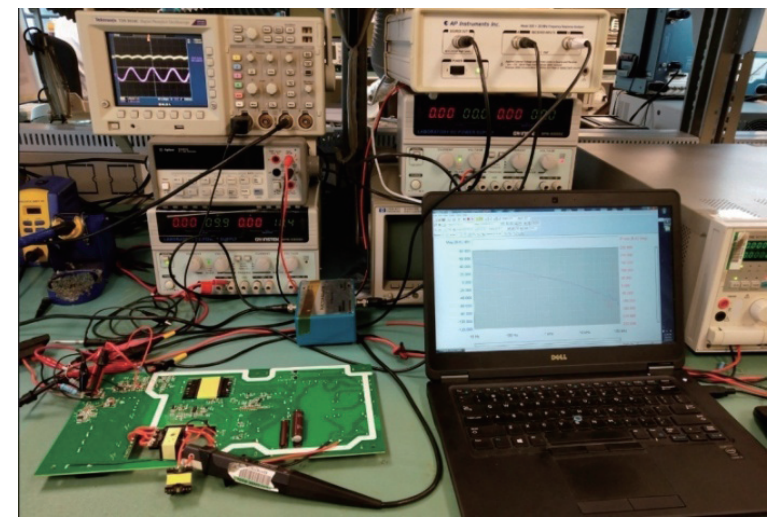

Fig. 22. The loop verification platform based on a TV power board.

TABLE III

PARAMETERS OF LLC CONVERTER FOR THE EXPERIMENT

\begin{tabular}{lcc}
\hline \hline Parameter & Description & Value \\
\hline$V_{\text {in }}$ & Nominal input PFC voltage [VDC] & 398 \\
$V_{\mathrm{o}}$ & Nominal output voltage [VDC] & 12 \\
$I_{\mathrm{o}-\text { max }}$ & Maximum output current [A] & 12 \\
$C_{\mathrm{s}}$ & Resonant Tank capacitor [nF] & 27 \\
$L_{\mathrm{s}}$ & Resonant Tank conductance [uH] & 107 \\
$L_{\mathrm{m}}$ & Magnetizing conductance [uH] & 840 \\
$N$ & Transformer ratio Np/Ns [uH] & $32 / 2$ \\
$C_{\text {divH }}$ & $U_{\mathrm{CS}}$ scaling capacitor (upside) [pF] & 82 \\
$C_{\text {divL }}$ & $U_{\mathrm{CS}}$ scaling capacitor (downside) [nF] & 8.2 \\
$C_{\mathrm{o}}$ & Output capacitor [uF] & $330 \times 5$ \\
$R_{c}$ & ESR of output capacitor [mR] & $28 / 5$ \\
CTR & CTR of OPTO & 0.4 \\
\hline \hline
\end{tabular}

the output load condition of 24 VDC\&9 A-15 A-15 A. It can be observed that the result of calculation matches well with the simulation result.

\section{EXPERIMENTAL VERIFICATION}

Shown as in Fig. 22, the proposed analytical small signal transfer functions regarding the open control to output loop and the closed overall loop can be verified further through the experiment on a $70^{\prime \prime} 250 \mathrm{~W} \mathrm{TV}$ power board by using UCC256304, in which, the output is 12 VDC\&12 A. The main design parameters are given in Table III.

Based on the dead time setting of $200 \mathrm{~ns}$, the operating frequency on the design board is: $94.3 \mathrm{kHz}$ for $2 \mathrm{~A}, 90.5 \mathrm{kHz}$ for $4 \mathrm{~A}, 89.6 \mathrm{kHz}$ for $5 \mathrm{~A}, 88.5 \mathrm{kHz}$ for $6 \mathrm{~A}, 86.5 \mathrm{kHz}$ for $8 \mathrm{~A}$ and $86.3 \mathrm{kHz}$ for $10 \mathrm{~A}$. The steady operation waveforms are shown in Fig. 23, in which the output ripple and the current though the resonant inductor are given. For the loop measurement, the AP instruments Model 300 is used as shown in Fig. 22. In order to obtain the plant loop, a $50 \mathrm{k} \Omega$ resistor is used to be in series with the lower output side of the OPTO FOD817A for the $V_{\mathrm{c}}$ pin measurement.

The open loop of power plant stage can be measured from UCC256304's FB voltage, which is actually located on the terminal of the added $50 \mathrm{k} \Omega$ resistor from the lower output side of FOD817A. Fig. 24 shows the plot comparison results between the measurements and the calculations when it
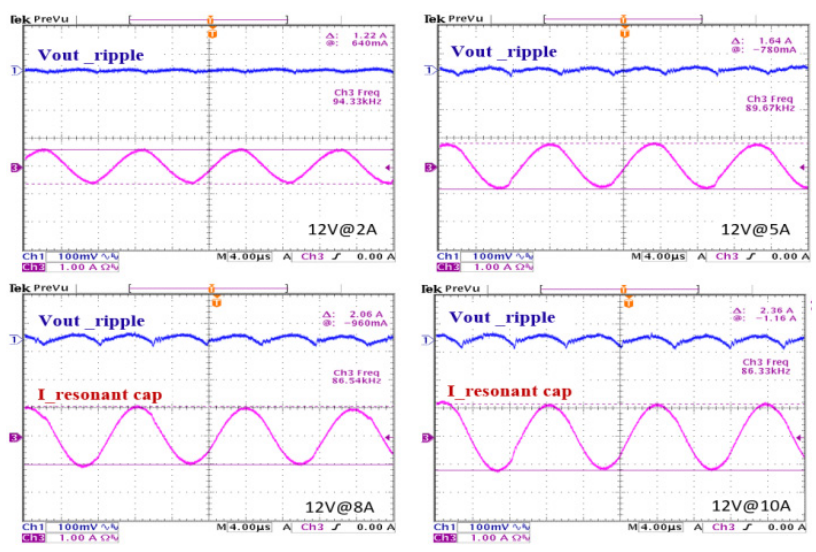

Fig. 23. Waveforms of output ripple and current of resonant tank under the output condition of 12 VDC@2 A, 5 A, 8 A and 10 A.
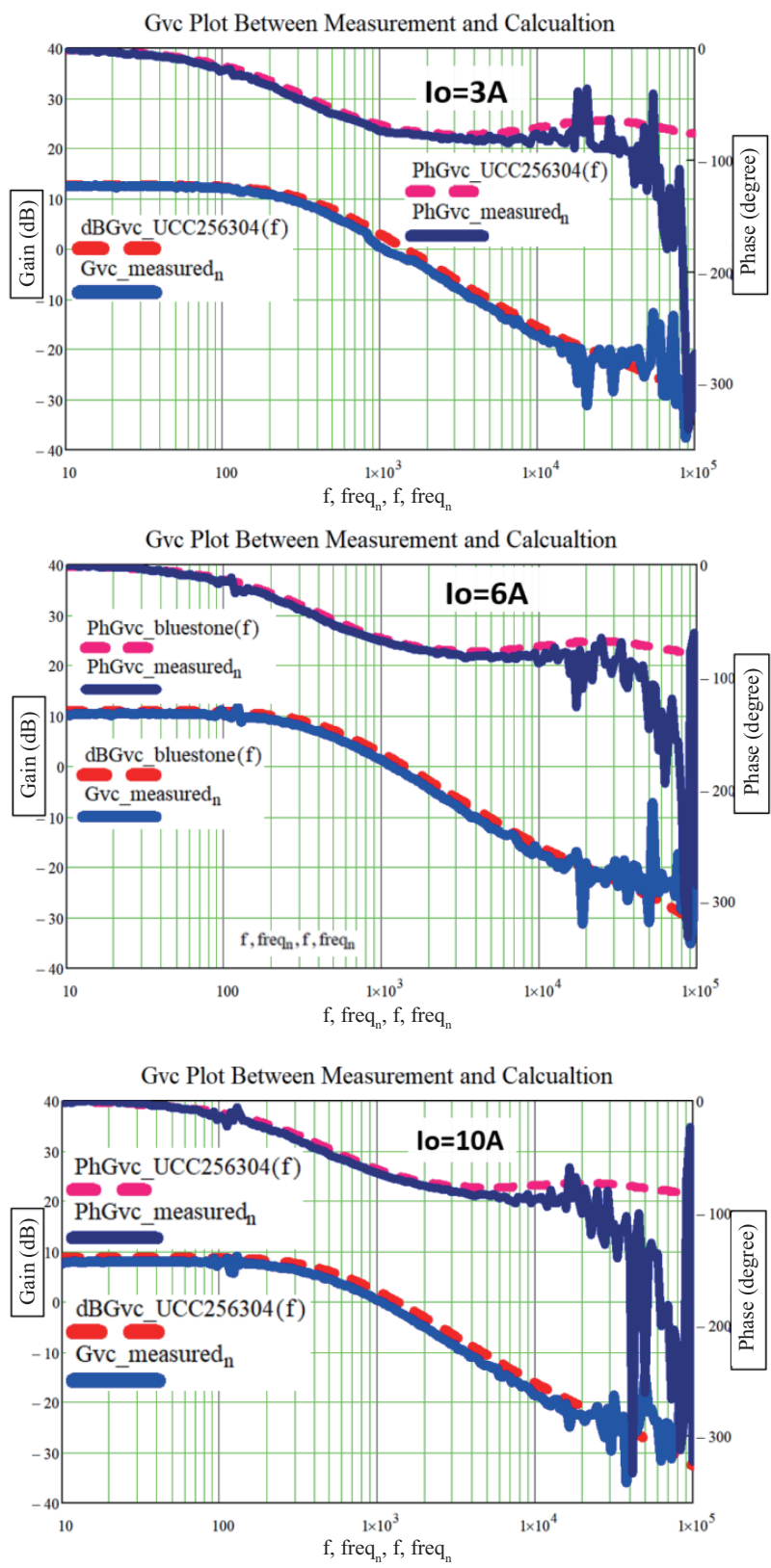

Fig. 24. Control to output plot comparison between measurement and calculation under the steady operation of $3 \mathrm{~A}, 6 \mathrm{~A}$ and $10 \mathrm{~A}$ output. 


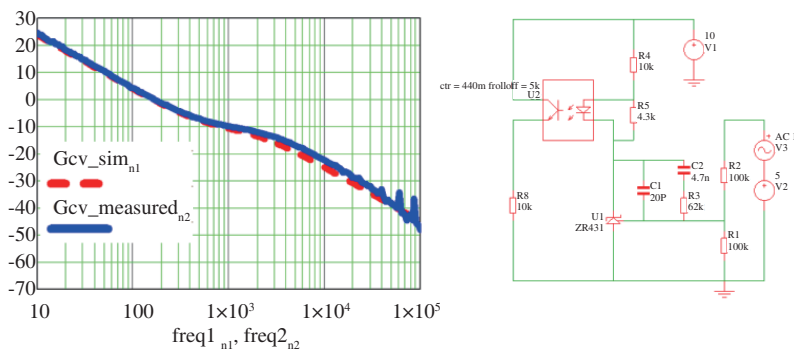

Fig. 25. Compensation plot comparison between simulation and measurement.

operates at $3 \mathrm{~A}, 6 \mathrm{~A}$ and $10 \mathrm{~A}$ respectively. The solid green lines represent the measurement results and the red dashed lines represent the calculation results, which show that these results match well practically. Though there may be concerns of dead time, it does not have impact from the prediction of the calculations if the frequency is given correctly. It is obvious to observe that the DC gain decreases with the increase of output current but the variation is relatively small compared to other hysteretic charge LLC converter. The cross frequency nearly remains the same result since the frequency variation is relatively very small.

To verify the transfer function of the overall loop, a practical precise model for the compensation circuit is required for this purpose because the transfer functions (23) is not with the consideration of the roll off frequency for FOD817A and TL431, which is necessary to obtain in practical measurement. Fig. 25 shows such a simulation example by pulling a $10 \mathrm{k} \Omega$ resistor at the emitter pin side of the opto-coupler for the rolling off frequency identification, in which the CTR is measured by 0.44 and the roll off frequency is verified to be $5 \mathrm{kHz}$. Refer to Fig. 15, with the other compensation parameters being set by $C_{\mathrm{v}}=4.7 \mathrm{nF}$, $C_{\mathrm{f}}=20 \mathrm{pF}, R_{\mathrm{v}}=62 \mathrm{k} \Omega, R_{\text {up }}=100 \mathrm{k} \Omega$ and $R_{\mathrm{f}}=10 \mathrm{k} \Omega$, the open loop plot of output to control can be obtained through simulation and measurement. Shown in Fig. 25, it can be seen the simulation result marked by the dashed red line matches well with the measurement result marked by the green solid line. With this consideration, a roll off pole is therefore added into (23) additionally, so the overall loop can be calculated precisely.

Fig. 26 shows the measurement results for the overall loop under the output of 12 VDC\&4 A and 12 VDC\& $10 \mathrm{~A}$ respectively, it can be observed both the cross frequency and phase margin are featured by a very small variation under the two kind of output conditions, which is a special and comforting result characterized by HHC control.

To verify the result of the calculation by (24), the comparison between calculation and measurement are obtained under the output of 12 VDC\&6 A. Shown in Fig. 27, the upside figure presents the measurement result and the downside figure shows the comparison under the output of $6 \mathrm{~A}$. It reveals that the measurement result marked by solid line match very well with the calculation result marked by a dashed line up to half of the operating frequency.

In order to verify the characteristic under dynamic load condition, the waveforms of output voltage and resonant
Overall Loop Test under 12VDC\&4A

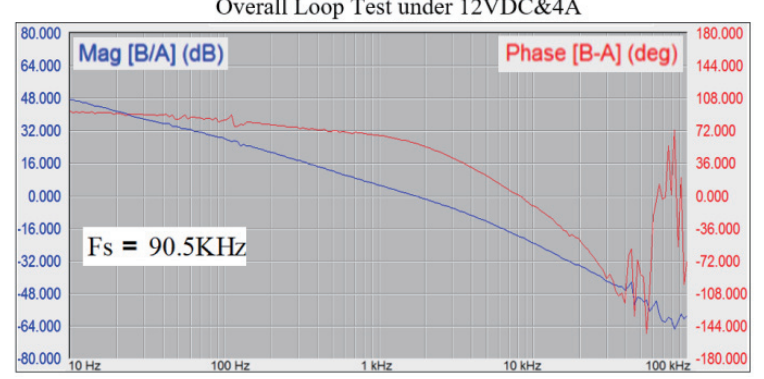

Overall Loop Test under 12VDC\&10A

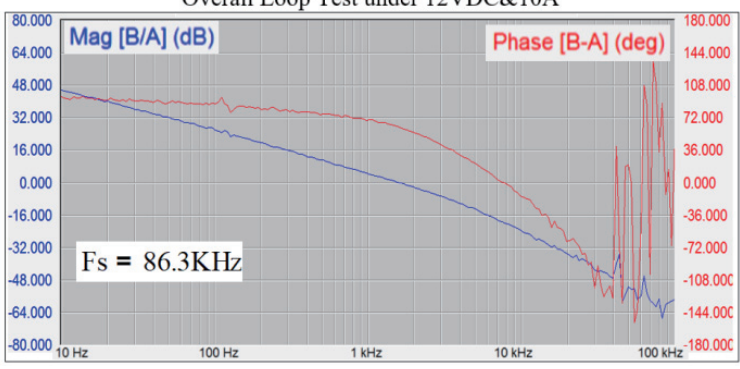

Fig. 26. Measurement results for the overall loop under the output condition of 12 VDC\&4 A (upside) and 12 VDC\&10 A (downside).
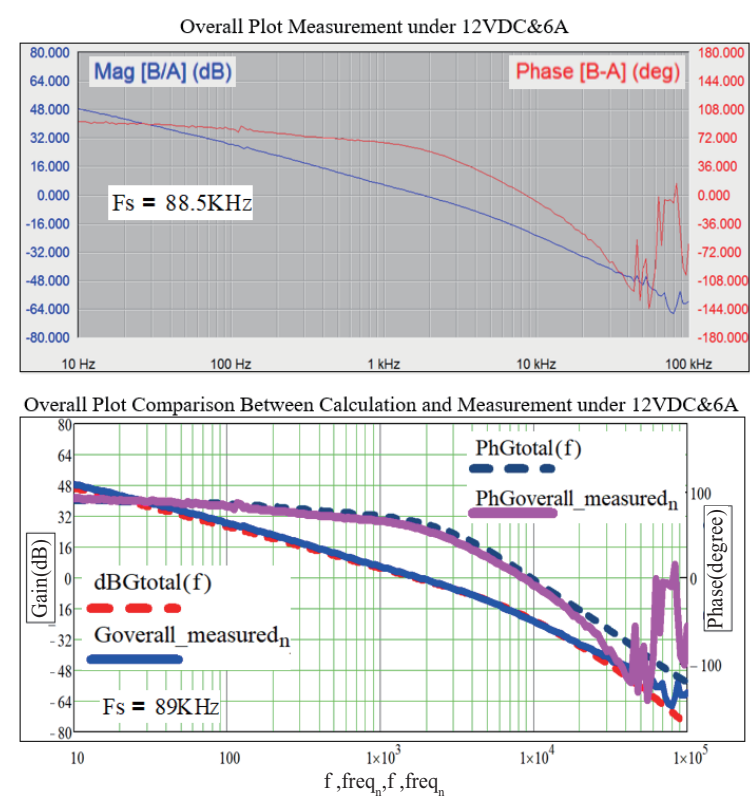

Fig. 27. Overall loop measurement under the output of 12 V\&6 A (on the topside) and the related comparison between the measurement and calculation (on the downside).

tank current are measured under the dynamic load condition of 12 VDC\&(4 A-8 A-4 A) and 12 VDC\&(8 A-12 A-8 A). Shown in Fig. 28, it can be observed the good dynamic performance can be achieved.

To verify the transfer function of closed output impedance given by (28), the dynamic load measurement is conducted based on a step current of 4 A- 8 A- $4 \mathrm{~A}$ with the slew rate setting by $3 \mathrm{~A} /$ us and step period-setting by $1 \mathrm{~ms}$. Fig. 29 shows such a comparison between calculation and measurement. It can be observed both results match well, which proves that the closed impendence is with good practical use for the practical design and applications based on LLC HHC control. 

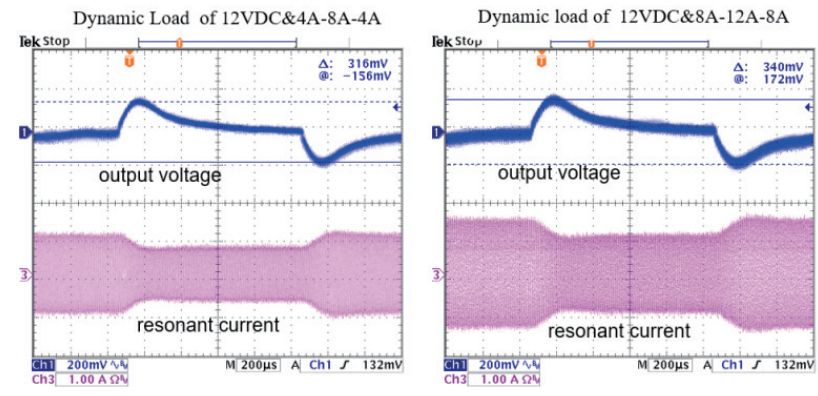

Fig. 28. Measurement of output voltage and resonant tank current

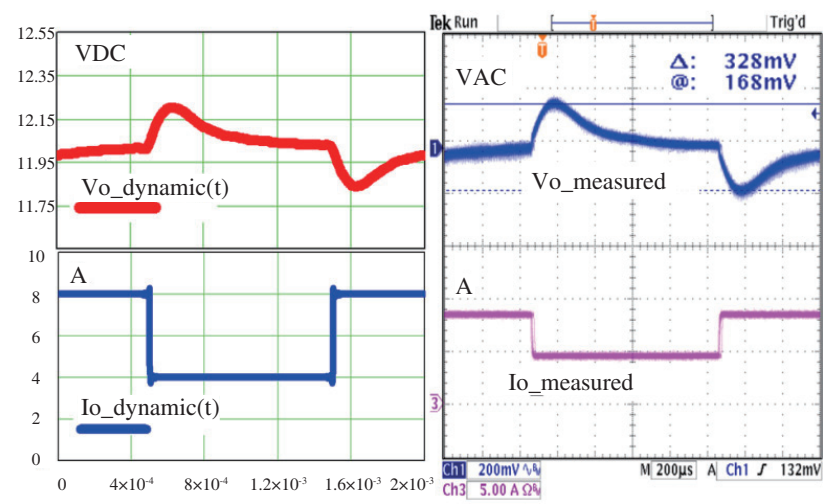

Fig. 29. Dynamic load response between calculation and measurement under the condition of 370 VDC input and 12 VDC\& 6 A output by 4 A-8 A-4A.

\section{CONCLUSIONS}

In this paper, the LLC HHC control has been introduced with the advantage of easy loop compensation and good dynamic characteristic compared to the conventional DFC control. The analytical small signal transfer functions applied for this control method have been therefore proposed and illustrated, in which the transfer functions of control to output, input to output, output impedance and the closed overall loop have been elaborated and verified. The overall comparison results based on the proposed transfer functions have been proved to match well with both the results from simulation and measurement in the most part of operating frequency range. More importantly, some physical insights related to the transfer functions are extracted and the design guideline for practical applications has been provided.

\section{REFERENCES}

[1] Bo Yang, F. C. Lee, A. J. Zhang, and Guisong Huang, "LLC resonant converter for front end DC/DC conversion," APEC. Seventeenth Annual IEEE Applied Power Electronics Conference and Exposition (Cat. No.02CH37335), Dallas, TX, USA, 2002, pp. 1108-1112, vol. 2.

[2] B. Yang and F. C. Lee, "Small-signal analysis of LLC Resonant Converter," in Proceedings of Center for Power Electronics System Seminar, pp. 1-6, 2003.

[3] Bing Lu, Wenduo Liu, Yan Liang, F. C. Lee, and J. D. van Wyk, "Optimal design methodology for LLC resonant converter," TwentyFirst Annual IEEE Applied Power Electronics Conference and Exposition, 2006. APEC '06., Dallas, TX, 2006, pp. 6 pp.-.

[4] C. Adragna, S. De Simone, and C. Spini, "A design methodology for LLC resonant converters based on inspection of resonant tank currents.," 2008 Twenty-Third Annual IEEE Applied Power Electronics Conference and Exposition, Austin, TX, 2008, pp. 1361-1367.

[5] X. Fang, H. Hu, J. Shen, and I. Batarseh, "An optimal design of the LLC resonant converter based on peak gain estimation," 2012 TwentySeventh Annual IEEE Applied Power Electronics Conference and Exposition (APEC), Orlando, FL, 2012, pp. 1286-1291.

[6] X. Fang et al., "Efficiency-oriented optimal design of the LLC resonant converter based on peak gain placement," in IEEE Transactions on Power Electronics, vol. 28, no. 5, pp. 2285-2296, May 2013.

[7] R. D. Middlebrook and S. Cuk, "A general unified approach to modelling switching-converter power stages," 1976 IEEE Power Electronics Specialists Conference, Cleveland, OH, 1976, pp. 18-34.

[8] R.Ridley, "A new continuous-time model for current mode control," $\mathrm{Ph} . D$. dissertation, Virginia Polytechinic Institute State Univerisity, Blackburg,Virginia, Nov. 1990.

[9] S. Tian, "Equivalent circuit model of high frequency PWM and resonant converters," Ph.D. dissertation, , Dept. Elect. Comput. Eng, Virginia Polytechnic Inst. State Univ., Blacksburg, VA, USA, Aug. 2015.

[10] R. Yang, T. Hung, and P. Wang, “An accurate small-signal model with peak current mode control applied for the Active clamp forward converter used in the constant conduction mode," 2014 International Power Electronics and Application Conference and Exposition, Shanghai, 2014, pp. 243-250.

[11] R. Yang, "A practical small signal model for the interleaved parallelseries active clamp forward converter with peak current control," 2018 IEEE International Power Electronics and Application Conference and Exposition (PEAC), Shenzhen, 2018, pp. 1-7.

[12] Y. Yan and F. C. Lee, "Unified three-terminal switch model for current mode controls," 2010 IEEE Energy Conversion Congress and Exposition, Atlanta, GA, 2010, pp. 1965-1972.

[13] S. Tian, F. C. Lee, J. Li, Q. Li, and P. Liu, "Equivalent circuit model of constant on-time current mode control with external ramp compensation," 2014 IEEE Energy Conversion Congress and Exposition (ECCE), Pittsburgh, PA, 2014, pp. 3747-3754.

[14] J. Li and F. C. Lee, "New modeling approach and equivalent circuit representation for current-mode control," in IEEE Transactions on Power Electronics, vol. 25, no. 5, pp. 1218-1230, May 2010.

[15] E. X. Yang, F. C. Lee, M. and M. Jovanovic, "Small-signal modeling of power electronic circuits by extended describing function concept," in Proceedings of Virginia Power Electronics Center Seminar, pp. 167-178, 1991, C. Buccella.

[16] E. X. Yang, "Extended describing function method for smallsignal modelingof resonant and multi-resonant converters," Ph.D. dissertation, Dept.Elect. Comput. Eng., Virginia Tech, Blacksburg, VA, USA, Feb. 1994.

[17] C. Chang, E. Chang, C. Cheng, H. Cheng, and S. Lin, "Small signal modeling of LLC resonant converters based on extended describing function," 2012 International Symposium on Computer, Consumer and Control, Taichung, 2012, pp. 365-368.

[18] C. Buccella, C. Cecati, H. Latafat, P. Pepe, and K. Razi, "Linearization of LLC resonant converter model based on extended describing function concept," 2013 IEEE International Workshop on Inteligent Energy Systems (IWIES), Vienna, 2013, pp. 131-136.

[19] B. Cheng, F. Musavi, and W. G. Dunford, "Novel small signal modeling and control of an LLC resonant converter," 2014 IEEE Applied Power Electronics Conference and Exposition - APEC 2014, Fort Worth, TX, 2014, pp. 2828-2834.

[20] J. Stahl, T. Hieke, C. Oeder, and T. Duerbaum, "Small signal analysis of the resonant LLC converter," 2013 IEEE ECCE Asia Downunder, Melbourne, VIC, 2013, pp. 25-30.

[21] J. Sun and H. Grotstollen, "Averaged modeling and analysis of resonant converters," Proceedings of IEEE Power Electronics Specialist Conference - PESC '93, Seattle, WA, USA, 1993, pp. 707-713.

[22] S. Tian, F. C. Lee, Q. Li, and B. Li, "Small-signal equivalent circuit model of series resonant converter," 2015 IEEE Energy Conversion Congress and Exposition (ECCE), Montreal, QC, 2015, pp. 172-179.

[23] S. Tian, F. C. Lee, and Q. Li, "Equivalent circuit modeling of LLC resonant converter," 2016 IEEE Applied Power Electronics 
Conference and Exposition (APEC), Long Beach, CA, 2016, pp. $1608-1615$.

[24] Y. Hsieh and F. C. Lee, "Modeling resonant converters in a rotating coordinate," 2017 IEEE Energy Conversion Congress and Exposition (ECCE), Cincinnati, OH, 2017, pp. 237-243.

[25] Y. Hsieh and F. C. Lee, "Accurate small-signal modeling of resonant converter based on perturbation on the state plane," 2018 IEEE Energy Conversion Congress and Exposition (ECCE), Portland, OR, 2018, pp. 6809-6816.

[26] J. Jang, M. Joung, S. Choi, Y. Choi, and B. Choi, "Current mode control for LLC series resonant dc-to-dc converters," 2011 TwentySixth Annual IEEE Applied Power Electronics Conference and Exposition (APEC), Fort Worth, TX, 2011, pp. 21-27.

[27] Jinhaeng Jang, Pidaparthy Syam Kumar, Dongyun Kim, and Byungcho Choi, "Average current-mode control for LLC series resonant dc-to-dc converters," Proceedings of The 7th International Power Electronics and Motion Control Conference, Harbin, 2012, pp. 923-930.

[28] H. Choi, "Charge current control for LLC resonant converter," 2015 IEEE Applied Power Electronics Conference and Exposition (APEC), Charlotte, NC, 2015, pp. 1448-1452.

[29] Z. Hu, Y. Liu, and P. C. Sen, "Bang-Bang Charge Control for LLC Resonant Converters," in IEEE Transactions on Power Electronics, vol. 30, no. 2, pp. 1093-1108, Feb. 2015.

[30] S. Kang and B. Cho, "Digitally implemented charge control for LLC resonant converters," in IEEE Transactions on Industrial Electronics, vol. 64, no. 8, pp. 6159-6168, Aug. 2017.

[31] Texas Instruments, "UCC256301 hybrid hysteretic mode wide Vin LLC resonant controller enabling ultra-low standby power," UCC256301 datasheet,Aug. 2017.

[32] B. McDonald and Y. Li, "A novel LLC resonant controller with bestin-class transient performance and low standby power consumption," 2018 IEEE Applied Power Electronics Conference and Exposition (APEC), San Antonio, TX, 2018, pp. 489-493.

[33] R. Yang, B. McDonald, and Y. Li, "A practical analytical small signal mode applied for the LLC converter based on hybrid hysteretic charge control," 2018 IEEE International Power Electronics and Application Conference and Exposition (PEAC), Shenzhen, 2018, pp. 1-8.

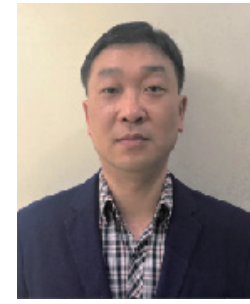

Richard (Hua) Yang received the B.S. and M.S. degrees from Hubei University of technology and South China University of Technology in electrical engineering in 2001 and 2014 respectively. From 2003 to 2011 , he was a senior power design engineer and technologist in Vapel and Emerson Network involved in the design of telecom\&industry power products.

In 2011, he joined Texas Instrumens China as senior Power and Analog application engineer responsible for the application of products solution in consumer, industry and enterprise computing. In power electronic area, he authored over ten papers in TI and IEEE conference and journals, with the experiences of research on popular power solutions and paper peer review on IEEE conference and transactions, his interest includes soft-switching $\mathrm{AC} / \mathrm{DC}$ and $\mathrm{DC} / \mathrm{DC}$ converter, voltage regulator modules, control, modeling and simulations. He was a recipient of the 2018 IEEE International Power Electronics and Application Conference and Exposition (PEAC) Excellent Paper Award.

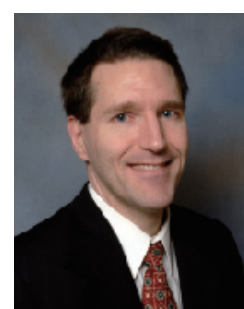

Brent A. McDonald received the B.S. degree in electrical engineering from the University of Wisconsin, Milwaukee, in 1996 and the M.S.E.E. degree from the University of Colorado, Boulder, in 2007. From 1996 to 2000, he was involved in the research and development in the power conversion group at Rockwell Collins in Cedar Rapids, IA. He was involved in the design and research of new power conversion techniques for avionics applications. From 2000 to 2008, he was a Power Conversion Technologist at Dell Inc. in Austin, TX, where he was involved in the design of dc-dc converters for desktops, notebooks, and servers. In addition, he provided five-year roadmaps, predicting power conversion trends for the computer industry. Currently, he is a System Architect defining next generation power controllers at Texas Instruments in Dallas, TX.

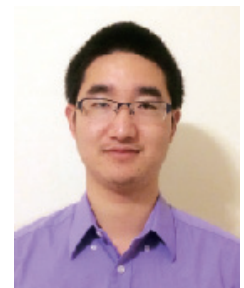

Yalong Li received the B.S. degree from Huazhong University of Science and Technology, Wuhan, China, in 2011. He received the M.S. and Ph.D. degree from the University of Tennessee, Knoxville, TN, USA, in 2013 and 2016, respectively, both in electrical engineering.

He was a Research Assistant in Center for UltraWide-Area Resilient Electric Energy Transmission Networks (CURENT), University of Tennessee, Knoxville, TN, USA, from 2011 to 2016. He joined Texas Instruments, Dallas, TX, USA in 2016. His current research interests include high voltage $\mathrm{AC} / \mathrm{DC}$ control. 